\title{
Welt der Weltphilologie:
}

Die Briefe Uku Masings an Enno Littmann 1933-1943

\author{
Henning Trüper
}

Online publiziert: 29. November 2018

(C) Der/die Autor(en) 2018

Zusammenfassung Die neun erhaltenen Briefe des estnischen Philologen, Theologen, Philosophen und Lyrikers Uku Masing an seinen Lehrer, den Tübinger Semitisten Enno Littmann, zum überwiegenden Teil 1940-1943 geschrieben, bieten Einblick in die Frage, was bis Mitte des 20. Jahrhunderts unter dem Weltbezug von Weltphilologie verstanden wurde. Der Aufsatz kontrastiert Masings mit Erich Auerbachs Verständnis von »Weltphilologie« von 1953 und verfolgt insbesondere, wie Auerbach und Masing geschichtsphilosophische Signaturen nutzen, um ihre Auffassung philologischer Praxis im Zeichen eines universalisierten Exils bzw. der Bezeugung einer umfassenden Verletzlichkeit der Gelehrsamkeit zu fomulieren. Im Anschluss an diese Diskussion folgt eine kommentierte Edition der Briefe.

\section{The World of World Philology}

Uku Masing's Letters to Enno Littmann 1933-1943

Abstract The nine surviving letters by the Estonian philologist, theologian, philosopher and lyricist Uku Masing to his teacher, the Tübingen professor of Semitic philology Enno Littmann, for the most part written 1940-1943, offer insight into the question of what, until the mid-20th century, was meant by »world « in the context of world philology. The article contrasts Masing's position with Erich Auerbach's 1950s notion of »world philology«. By means of this comparison, the radical nature of Masing's understanding of philological practice can be grasped. The article discusses the manner in which Auerbach and Masing apply notions from the philosophy

Für Gegenlektüre und philologischen Beistand danke ich Kaj Öhrnberg, Mario Wimmer und Ilkka Lindstedt.

H. Trüper $(\bowtie)$

Helsinki Collegium for Advanced Studies, University of Helsinki,

Fabianinkatu 24, P.O. Box 4, 00014 Helsinki, Finnland

E-Mail: henning.trueper@gmail.com 
of history to philological practice, which they understand as a universalization of exile and as testimony to the comprehensive vulnerability of scholarship respectively. This discussion is followed by an annotated edition of the letters.

\section{I.}

Die Korrespondenz, die der estnische Philologe, Theologe, Philosoph und Lyriker Uku Masing (1909-1984) an den deutschen Semitisten Enno Littmann (1875-1958) richtete, umfasst nicht mehr als neun erhaltene Briefe. ${ }^{1}$ Diese kleine Sammlung, die hier eingeleitet, ediert und erläutert wird, gibt Auskunft, nicht allein über einige, übrigens nicht unwichtige Episoden aus dem etwas rastlosen Verstandesleben ihres Verfassers, sondern auch über die Beschaffenheit eines über den betroffenen Briefschreiber weit hinausreichenden »weltphilologischen« Interesses in der Mitte des 20. Jahrhunderts. Dieses Interesse besser zu verstehen vermehrt das Verständnis der Geschichte der Philologien und der umgebenden Geisteswissenschaften.

»Weltphilologie« ist ein Begriff, den Erich Auerbach 1952 eher beiläufig aufbrachte, als er über das Problem einer »Philologie der Weltliteratur « nachdachte. ${ }^{2}$ Die Begriffsbildung folgt dem älteren, Goethe'schen Begriff der »Weltliteratur« (erläutert etwa in der berühmten Einlassung vom 31. Januar 1827 in den Gesprächen mit Eckermann) ${ }^{3}$ und verpflichtet das literarische Studium (denn dieses ist bei Auerbach vorzüglich gemeint) auf Anerkennung der weltweiten Verbundenheit der Texte, Stoffe und Formen. Über das Problem der Weltliteratur ist in den letzten Jahren viel geschrieben worden, etwa in Hinsicht auf globale Kanonbildung, Marktgängigkeit, ästhetische Unübersetzbarkeit und kolonial induzierte Ungleichgewichte. ${ }^{4}$ Über das verwandte Problem der Weltphilologie ist dagegen bislang vergleichsweise weniger gearbeitet worden, wenn auch einige wichtige Arbeiten zu diesem Komplex erschie-

\footnotetext{
1 Staatsbibliothek Berlin, Handschriftenabteilung, Nachlass 245 (Enno Littmann), K. 20. Zu Masing, der eine bedeutende, international allerdings mangels Übersetzungen seiner wichtigeren theologischen, philosophischen und literarischen Arbeiten wenig rezipierte Figur der estnischen Geistes- und Literaturgeschichte ist, siehe Vincent B. Leitch, »Religious Vision in Modern Poetry: Uku Masing Compared with Hopkins and Elliot«, Journal of Baltic Studies 5 (1974), 281-294; Külliki Kuusk, »Uku Masing: Stretching the Borders«, Estonian Literary Magazine 28 (spring), http://elm.estinst.ee/issue/28/uku-masingstretching-borders/ [5.6.2017]; Urmas Nõmmik, »Uku Masing und das Alte Testament«, in: Tarmo Kulmar, Rüdiger Schmitt (Hrsg.), Ideas of Man in the Conceptions of the Religions. Akten des VIII. gemeinsamen Symposiums der Theologischen Fakultät der Universität Tartu, der Deutschen Religionsgeschichtlichen Studiengesellschaft und der Estnischen Studiengesellschaft für Morgenlandkunde und der Studiengesellschaft für Theologie am 2. und 3. Oktober 2009 an der Universität Tartu/Estland, Münster 2012, 187-198.

2 Erich Auerbach, »Philologie der Weltliteratur«, in: Walter Muschg, Emil Staiger (Hrsg.), Weltliteratur: Festgabe für Fritz Strich zum 70. Geburtstag, Bern 1952, 39-50.

3 Johann Peter Eckermann, Gespräche mit Goethe in den letzten Jahren seines Lebens, Frankfurter Ausgabe II, 39, Frankfurt a.M. 1999.

4 Vgl. besonders Pascale Casanova, La République mondiale des lettres, Paris 1999; David Damrosch, What is World Literature?, Princeton 2003; Emily Apter, Against World Literature: On the Politics of Untranslatability, London 2013.
} 
nen sind. ${ }^{5}$ Verwandt sind diese Probleme unter anderem deswegen, weil sowohl der modernen Literatur als auch der modernen Philologie der Impuls zur Theoriebildung inhärent ist, sodass ein nur schwer umgehbarer Überschneidungsbereich entsteht.

In Umdeutung des Wilamowitz'schen Schmähworts von der »Zukunftsphilologie« ist in den letzten Jahren die Frage nach der künftigen methodischen Ausrichtung der Textwissenschaften neu aufgeworfen worden. ${ }^{6}$ Es ist wohl nicht falsch, wenn man den Begriff der Philologie, der sich in diesem Zusammenhang neu formiert hat, als Bezeichnung eines zwar vielfältigen, jedoch klar umgrenzten Methodenraums auffasst, der als eine Art Forum für eine künftige, global ausgerichtete geisteswissenschaftliche Arbeit dienen soll. Von der geschäftigen Frequentierung dieses Forums erhofft man sich zugleich eine Absicherung der Anerkennung solcher Forschung durch politische und gesellschaftliche Instanzen. Es wäre dann allerdings anzumerken, dass dieses Programm nicht unabhängig von der Frage entwickelt werden kann, welche Bausubstanz denn am Ort schon vorhanden ist (und seien es bloß Ruinen). Solange man nicht ganz jenseits des Topos der Philologie neu baut - und natürlich ist dies in den Jahrzehnten der versuchten Abkehr von der Philologie unternommen worden, nur sind die entstandenen Betonwüsten anscheinend auch nicht wohnlicher -, prägen die alten Bedeutungsrelikte die Gegend.

Analog gilt auch für den Begriff des »Globalen«, der für eine Zukunft jenseits des Eurozentrismus nicht wegzudenken wäre, dass er sich mit vorgängigen Auslegungen des Begriffs von Welt in der Philologie auseinandersetzen sollte. Ansonsten liefe man wohl Gefahr, sich unversehens in Abhängigkeit von Metaphern wie jenen des Weltmarkts und der Weltwirtschaft wiederzufinden. Kurz, je gegenwärtiger das Interesse am Philologischen ist, desto mehr gewinnt auch die Philologiegeschichte an Bedeutung. Es ist unabdingbar, dass diese historische Forschung aus ihrer isolierteuropäischen Perspektive heraustritt, indem sie etwa textwissenschaftliche Traditionen auch in anderen Weltgegenden untersucht und zum Beispiel nachvollzieht, wie die »westliche« Rezeption derartige Traditionen zugunsten einer ausschließlichen Lektüre der Urtexte vernachlässigt hat, wie Sheldon Pollocks Beiträge betonen. Ein solches Heraustreten müsste zugleich eine Revision gängiger Auffassungen der Geschichte textwissenschaftlicher Bemühungen in Europa nach sich ziehen. Diese Revision könnte sich nicht darauf beschränken, einmal mehr die politischen Nutzungen der Philologien im Hinblick auf Nationalismus, Rassismus, Antisemitismus und Imperialismus zu destruieren und sich danach auf der Grundlage eines vermeintlich stabilen und unbeschadet gebliebenen methodischen Unterbaus wieder neu einzurichten. Vielmehr sollte das Verständnis der Beschaffenheit dieses Unterbaus selbst revidiert werden. Aus diesem Grund ist auch eine genauere Untersuchung der historischen Auffassungen des philologischen Begriffs von »Welt« wünschenswert; und hierfür sind nun eben die Masing'schen Briefe eine hilfreiche Quelle.

\footnotetext{
5 Vgl. besonders Sheldon Pollock, »Philology and Freedom«, Philological Encounters 1 (2016), 4-30 und Islam Dayeh, »The Potential of World Philology«, Philological Encounters 1 (2016), 396-418, ferner Sheldon Pollock, Benjamin A. Elman, Ku-ming Kevin Chang (Hrsg.), World Philology, Cambridge, MA 2015.

6 Vgl. den Forschungsverbund Zukunftsphilologie am Forum transregionale Studien der Freien Universität Berlin und die dort begründete neue Zeitschrift Philological Encounters (Leiden), http://www.forumtransregionale-studien.de/programme-und-initiativen/zukunftsphilologie.html [13.10.2017].
} 


\section{II.}

Für die historische Frage nach der Welt von Weltphilologie bieten Auerbachs weitere Angaben zur Auslegung des Begriffs in seinem Festschriftaufsatz von 1952 nach wie vor einen, vielleicht sogar den bestgeeigneten Ansatzpunkt:

Die mit wissenschaftlichen Methoden betriebene Erforschung der Weltwirklichkeit erfüllt und beherrscht unser Leben; sie ist, wenn man so will, unser Mythos; denn einen anderen, der allgemein Gültigkeit hätte, besitzen wir nicht. Innerhalb der Weltwirklichkeit ist die Geschichte dasjenige, was uns am unmittelbarsten trifft [...]. Denn sie ist der einzige Gegenstand, in welchem die Menschen im Ganzen vor uns treten. [...] Die innere Geschichte der letzten Jahrtausende, welche die Philologie als historische Disziplin behandelt, ist die Geschichte der zum Selbstausdruck gelangten Menschheit. ${ }^{7}$

Auerbach führt weiter aus, dieses historische Studium des menschheitlichen Selbstausdrucks befinde sich nach Kriegsende in einer Lage besonderer Begünstigung, eines Kairos, in dem durch die Konjunktion historischer Bildungstraditionen und zeitgenössischer Erfahrung ein ungewöhnliches Maß an »historisch-perspektivischem Sinn $\ll^{8} »$ noch $«$ gegeben sei. Dieser Kairos der Geschichtsschreibung wird sodann weiter ausgelegt durch ein Zitat aus Adalbert Stifters Nachsommer: »Es wäre des höchsten Wunsches würdig, wenn nach Abschluß des Menschlichen ein Geist die gesamte Kunst des menschlichen Geschlechtes von ihrem Entstehen bis zu ihrem Vergehen zusammenfassen und überschauen dürfte. «9 Auerbach kommentiert, obwohl vom vollständigen »Abschluss « des menschlichen Wesens auch 1952 nicht die Rede sein könne, sei doch ein gewisser »Ort des Abschlusses und der Wendung « erreicht, »wie nie zuvor« (ebd.). Die mythische Aufladung der Philologie der Weltliteratur ist bedingt durch das Erreichen dieses historischen »Orts«, als Standpunkt eines weltumfassenden Bewusstseins auf dem Höhepunkt einer solchermaßen an ihr Ende gelangten Entwicklung. Hegels »Ende der Kunstperiode « wäre mitzudenken. ${ }^{10}$ Auerbachs Hinweise zur Bedeutung von »Welt« zielen also auf das Historische in geschichtsphilosophischem Sinn: Entwicklung, Ende (der Kunst), Ganzheit. Der Philologie kommt aus Auerbachs Sicht das Privileg zu, die »innere« Geschichte der Menschheit offenzulegen, also mit der klassischen Formel August Boeckhs: die »Erkenntnis des Erkannten«. Im Hiat nach dem Ende des Zweiten Weltkriegs, nach dem Zusammenbruch der europäischen Moderne seien die Philologen in die Lage versetzt, einen umfassenden menschheitlichen Rahmen zu überschauen. Sie hätten Gelegenheit, zu Weltphilologen zu werden.

\footnotetext{
7 Auerbach (Anm. 2), 41.

8 Auerbach (Anm. 2), 41. Zu diesem Topos vgl. Reinhart Koselleck, »Standortbindung und Zeitlichkeit: Ein Beitrag zur historiografischen Erschließung der geschichtlichen Welt«, in: Ders., Vergangene Zukunft: Zur Semantik geschichtlicher Zeiten, Frankfurt a.M. 1979, 176-207.

9 Zitiert nach Auerbach (Anm. 2), 42.

10 Eva Geulen, Das Ende der Kunst: Lesarten eines Gerüchts nach Hegel, Frankfurt a.M. 2002.
} 
In der gegenwärtigen Debatte über die unerledigte Geschichte der Philologie, möglicherweise sogar ihre Wiederkehr (oder die Rückkehr zu ihr) sowie ihre Zukunft wird Auerbachs Findung gelegentlich als Chiffre für eine kosmopolitische Utopie aufgefasst, als Idealisierung eines Forschungsprozesses, in dem die Daseinsbedingung des Exils gewissermaßen universalisiert werde. ${ }^{11}$ In einem solchen allgemeinen Exil würden die partikularen Bindungen der »inneren Geschichte« der Menschheit an Zeiten und Räume aufgehoben. Die Subtilität der Auerbach'schen Bestimmung des Aufgabenbereichs des kommenden »Weltphilologen « liegt jedoch noch anderswo, nämlich in der Annahme, die moderne Wissenschaft (als einheitliches System des expandierenden Vernunftgebrauchs) befinde sich in der Lage einer eskalierenden mythischen Verstricktheit. Es ist diese Annahme, die in jenem kurzen Festschriftaufsatz zwischen dem scheinbar ungebrochen optimistischen Wissenschaftsverständnis einerseits und der auf die Figur des Endes ausgerichteten geschichtsphilosophischen Aufladung andererseits vermittelt. Der wissenschaftliche und technische Fortschritt bedingt nämlich zugleich ein beschleunigtes Wuchern des Mythos. Die »Welt« der Philologie bleibt auf die Erfahrung der Einzelnen gegründet, deren tiefe Unverständlichkeit in der wissenschaftlichen Arbeit nicht aufgehoben, nur verwandelt wird.

Dennoch kommt diese Konstruktion nicht umhin, die Gegenwart vor der Vergangenheit auszuzeichnen, schon weil das Jetzt eben näher an der Zukunft liegt als das Vergangene. Entsprechend verkennt oder unterschlägt Auerbachs Entwurf einer Weltphilologie das Vorhandensein einer entsprechenden Forschungspraxis - einer Philologie der allgemeinen globalen Verbundenheit des überkommenen Textbestands - in den Geisteswissenschaften des 19. und frühen 20. Jahrhunderts. Willentlich oder unwillkürlich nimmt Auerbach eine geschichtsphilosophische Überschreibung des gegebenen Bestands vor. Die Erklärung für diesen Akt der Überschreibung ist wohl mindestens zum Teil in einer Tradition der Marginalisierung und Nichtkanonisierung zu finden, die innerhalb der Fächerfamilie der Philologien besonders bestimmte Formen der Orientalistik betraf und sich vor allem im Hinblick auf die Spielarten des Antikenbezugs entwickelte. Auerbachs eigene literaturgeschichtliche Sichtweise, in der die Existenz einer kontinuierlichen und vergleichsweise autonomen europäischen Tradition mit zweifachem Ursprung in Athen und Jerusalem geradezu als Voraussetzung fungiert, ist selbst ein Beispiel für die hergebrachten Argumentationsfiguren des Antikenbezugs. Wohl war, wie James Porter gezeigt hat, Auerbachs Umsetzung des Gemeinplatzes von der Konkurrenz von Griechen und Hebräern anti-hegemonial, weil sie nicht für den Vorrang Athens, sondern den Jerusalems eintrat. ${ }^{12}$ Doch hatte über das gesamte 19. Jahrhundert hinweg und bis weit ins 20. neben anderen auch eine Forschungsrichtung bestanden, die abweichende Vergangenheitsbezüge europäischer Traditionen nachweisen wollte. Die impliziten Antagonismen wurden in diesem Forschungszusammenhang aufgegeben und durch eine Geschichte von Kulturtransfers (»Entlehnungen« im Zeitgebrauch) innerhalb einer als einheitlich und verflochten verstandenen Alten Welt ersetzt. Zugleich wurde die Konkurrenz erweitert, sodass etwa auch Altägypten, das vedische Indien,

\footnotetext{
11 Insbesondere Edward Saids Bezugnahmen auf Auerbach lassen sich so lesen, z. B. in »The Return to Philology«, in: Humanism and Democratic Criticism, New York 2004, 57-84.

12 James Porter, »Erich Auerbach and the Judaizing of Philology«, Critical Inquiry 35/1 (2008), 115-147.
} 
Babylonien, Persien oder China in ein agonales Verhältnis zum klassisch geprägten Okzident eintreten konnten (während die hebräische Antike üblicherweise zugunsten der ägyptischen und babylonischen Nachbarn marginalisiert wurde). Die fragliche Forschungstradition war von gegenläufigen Tendenzen geprägt, nämlich einerseits zur Aufhebung, andererseits zur unkontrollierten Erweiterung der Vorstellung selbstgenügsamer »Hochkulturen« mit konkurrenzfähiger Wertigkeit. Aber innerhalb dieses Rahmens war die Hybridisierung der europäischen Geschichte mit ihren nichteuropäischen kulturgeschichtlichen Umgebungen prominentes Forschungsziel einer bestimmten Traditionslinie besonders der Orientalistik. ${ }^{13}$

Beispiele für derartige Perspektiven lassen sich zahlreich nachweisen; und Kategorien lassen sich ohne größeren Aufwand formulieren: Man studierte erstens die Fernwirkung »orientalischer« Antiken wie der ägyptischen, indischen und persischen (Friedrich Creuzer kann hier als Zentralfall schon aus der romantischen Ära gelten), späterhin auch der babylonischen oder chinesischen; zweitens die Nahwirkung kultureller Beziehungen im Mittelmeerraum und unter dessen Vermittlung (so in den Arbeiten über die vermuteten islamischen Quellen Dantes; über das Schattentheater, das die Orientalisten der Zeit um 1910 besonders interessierte; oder die Technologietransfers der Seidenstraße); und drittens auch den vormodernen Transfer europäischer Traditionen in außereuropäische Gebiete (so die Arbeiten über den Transfer katholischer Marienlegenden nach Äthiopien im 15. und 16. Jahrhundert, zu denen Masing beitragen wollte). ${ }^{14}$

So gesehen ist es verwunderlich, dass Auerbach offenbar meinte, eine »weltliterarisch« orientierte Philologie sei noch gar nicht auch nur im Ansatz unternommen worden. Weniger verwunderlich ist dieser Umstand, wenn man sich vor Augen hält, dass schon seit dem Streit zwischen Boeckh und Hermann in den 1820er Jahren die Altertumswissenschaft ausdrücklich darauf beharrte, einen vorgeblich selbstgenügsamen griechisch-römischen Kernbereich zu privilegieren. Dieses klassizistische Beharren wurde zum übermächtig wirksamen Imperativ der Philologien insgesamt, weil sich auch die mediävistischen und neusprachlichen Fachgebiete - in denen etwa der Romanist Auerbach ausgebildet war - vielfach diesem Gebot unterwarfen. Den neuphilologischen Fächern bot der altphilologische Klassizismus einerseits eine Rückversicherung über die ansonsten stets fragwürdige Einheit der europäischen Literatur; und andererseits die Garantie der Wertigkeit des eigenen Forschungsgegenstands als Zielhafen der literarischen Traditionen der griechischen Antike, die im Bildungssystem nach wie vor als normativ galten. Den Gewohnheiten der Privilegierung bestimmter Bereiche korrelierten Gewohnheiten der Marginalisierung anderer.

\footnotetext{
13 Worauf insbesondere Suzanne Marchand, German Orientalism in the Age of Empire: Religion, Race, and Scholarship, Cambridge 2009 hingewiesen hat.

14 Vgl. zu diesem Komplex Andrea Celli, Dante e l'Oriente: Le fonti islamiche nella storiografia novecentesca, Rom 2013, hier Kapitel 1 über Enrico Cerulli. Zu Cerulli siehe auch Karla Mallette, European Modernity and the Arab Mediterranean: Toward a New Philology and a Counter-Orientalism, Philadelphia 2010, Kap. 5.
} 
Die Forschungsarbeiten und Briefe Masings bieten dem philologiegeschichtlichen Interesse Einblick in eine entsprechende und übrigens tiefe thematische, geografische und institutionelle Marginalität. Mithilfe eines solchen Einblicks können erstens die Vorstellungen hinter der Auerbach'schen Formel von der Weltphilologie weiter untersucht werden, zweitens aber auch die spezifischen Gegnerschaften innerhalb der weltphilologischen Arbeit verständlicher gemacht werden: Der jeweilige Weltbegriff prägte sich jeweils gegen andere Forschungsrichtungen aus, nicht allein in inhaltlicher Hinsicht, sondern auch in institutionellen, geografischen und sprachlichen. Die Provinzialität eines Dokumentenbestandes wie desjenigen der Masing'schen Briefe hilft dabei, diese Kondition der Gegnerschaft sichtbarer zu machen als es vielleicht anderwärts der Fall wäre. Die Weltphilologie ist schon von vornherein zerstreut in jenem Raum, zu dem sie behauptet, überhaupt erst Zugang zu gewinnen. Das Problem des Exils ist in der Philologiegeschichte eng mit diesem Umstand verbunden, insofern es selbst eine Bedingung der räumlichen Zerstreuung und der erzwungenen Marginalisierung bildet. Als epistemologische Situation, die es ja im zukunftsphilologischen Verständnis sein soll, ist es an eine gegnerschaftliche Auslegung der Welt von Weltphilologie gebunden, die man besser vom Rand her studieren kann.

\section{III.}

Die Briefe Masings an Littmann, die mit Ausnahme des ersten Schreibens von 1933 aus den Jahren 1940-43 stammen, markieren die Fallhöhe im Weltbezug der Philologie. Masing hatte Anfang der dreißiger Jahre zuerst in Tübingen bei Littmann und dann bei verschiedenen Semitisten und Theologen in Berlin studiert. Dass er sich in der Folge bemühte, mit einem - vielleicht übrigens auch noch anderen - seiner Lehrer Kontakt zu halten, mag einem Interesse am Zugang zu Büchern und Publikationsmöglichkeiten geschuldet gewesen sein, wie es in den Briefen mehrfach zur Sprache kommt, auch wohl einem Gebrauch der Verehrung akademischer Lehrer, der für die Briefrhetorik dieser Periode prägend war. Allerdings fällt der Hauptteil der kleinen Korrespondenz in die Zeit der größten politischen Unsicherheit. Deswegen ist es wohl nicht abwegig anzunehmen, dass sich Masing durch einen Briefwechsel wie den mit Littmann erhofft haben dürfte, in der Zeit nach einer deutschen Invasion falls nötig gegenüber den Repräsentanten der Besatzungsmacht auf einen institutionell gewichtigen Kontakt in Deutschland verweisen zu können. Littmann war nirgends besonders als Parteigänger des nationalsozialistischen Staatsund Wissenschaftswesens aufgefallen und hielt sich mit politischen Stellungnahmen zurück. ${ }^{15}$ Masing, der die Veränderung der Verhältnisse 1933 aus der Nähe hatte verfolgen können, lehnte die nationalsozialistische Ideologie und Herrschaft ent-

15 Vgl. allgemein zur Fachgeschichte Ekkehard Ellinger, Deutsche Orientalistik zur Zeit des Nationalsozialismus 1933-1945, Edingen-Neckarhausen 2006. Detaillierte Studien zu Individuen wie Littmann liegen kaum vor; die Interpretation auf ein geschlossenes ideologisches System hin, wie sie Ellinger vorschlägt, ist in gewisser Weise problematisch, obwohl der kritische Impuls einer solchen Lesart unerlässlich bleibt. Littmann, nach 1918 deutschnational orientiert, lässt sich kaum als Gegner des NS ansehen, kritisierte aber z. B. in Veröffentlichungen die in der italienischen Äthiopistik virulent werdende Bemühung, die äthiopischen Sprachen aus der semitischen Sprachfamilie auszugliedern und damit in antisemitisch-rassistischer Sicht aufzuwerten. Anders als im Ersten Weltkrieg unterließ es Littmann nach 1939, sich intensiv um die 
schieden ab. Vermutlich betrachtete er Littmann nicht als Parteigänger des Regimes. Nach Kriegsende wurde die Korrespondenz allerdings nicht fortgesetzt, vermutlich zunächst in Folge abgebrochener Postverhältnisse, wahrscheinlich aber auch, weil die Briefe ihre instrumentelle Funktion verloren hatten.

Trotz ihrer möglichen politisch-pragmatischen Lesbarkeit sind die Schreiben Masings auch Ausdruck eines feineren Sensoriums für die Frage nach dem Sitz der Gelehrsamkeit im Leben als man es bei Akademikern wohl zumeist antrifft. Im Zweiten Weltkrieg wird nun daraus die Frage nach dem Sitz der Wissenschaft im Sterben, die Masing andeutet, wenn er darauf hinweist (09.12.41), dass er seinen Unterhalt mit der Arbeit an der Katalogisierung von Büchern für die Universitätsbibliothek friste - denn »die jüdischen und hebräischen Bücher bleiben meistens für die Universität«, das heißt, nach der Ermordung ihrer vorherigen Besitzer im vorangegangenen Sommer - wie zum Beispiel des nach der Vertreibung von der Leipziger Universität 1933 nach Estland emigrierten Institutskollegen und, wie die Briefe nahelegen, zeitweiligen Konkurrenten Masings, Lazar Gulkowitsch. Seinen lettischen Studenten Isidor Levin (*1919) half Masing über die deutsche Invasion und die Massenerschießungen 1941 hinweg zu verstecken. ${ }^{16}$

Die sowjetische Invasion Estlands vom Sommer 1940 bespricht Masing in seinen Briefen nicht ausführlich. Beim Einmarsch der Deutschen im Juni 1941 tauchte er offenbar unter (09.12.41), zunächst wohl um der Einberufung in die Rote Armee oder möglichen Repressalien gegen die Universitätsangehörigen zu entgehen; aber vermutlich auch, um der Gewalt der neuen Besatzungsmacht auszuweichen. Schon vor der deutschen Invasion hatte Masing die Unruhe der »Nerven« mit medizinischen Mitteln zu unterdrücken versucht (14.05.41). Die Jahre der Ungewissheit über die Entwicklung der Universität hatten an dieser Schädigung der psychischen Gesundheit wohl ihren Teil, vielleicht aber auch die eingenommenen Medikamente selbst. Masing erwähnt en passant die Schließung der Theologischen Fakultät in der ersten sowjetischen Periode, ferner einen latenten Konflikt mit Gulkowitsch. Mehrfach berichtet er auch von anhaltender Ungewissheit über den Abschluss seiner Dissertation, genauer deren zweiten Teil, der zwar gedruckt, dann aber wieder eingestampft worden sei. Die Disputation des Gesamtwerks ließ sich trotz offenbar wiederholter Bemühungen um das Arrangement des Rituals nicht realisieren.

Warum unter diesen Umständen überhaupt Philologie? Zum einen hoffte Masing zunächst wohl, später wieder an der Universität arbeiten zu können, die ihn vor der ersten sowjetischen Invasion für semitische Philologie und Theologie des Alten Testaments beschäftigt hatte. Dieser Hoffnung entsprang vermutlich gerade auch sein Austritt aus der offenbar wiedereröffneten Theologischen Fakultät wenige Monate nach der deutschen Invasion. Denn wie auch die Hilfeleistung für Levin andeutet,

\footnotetext{
Nutzbarmachung seiner wissenschaftlichen Arbeit für den Krieg zu bemühen. Wie nicht ungewöhnlich, findet man auch unter seinen regelmäßigen Korrespondenzpartnern kein einheitliches politisches Profil.

16 Auf Veranlassung Levins wurden Masing (1969) und seine Frau Eha (1996) von der Gedenkstätte Yad Vashem geehrt: URL http://db.yadvashem.org/righteous/family.html?language=en\&itemId=4043741 [23.7.2017]. Levin wurde im März 1942 verhaftet, überlebte aber die diversen Gefängnisse und Konzentrationslager, in denen er inhaftiert war; Isidor Levin, »Geistige Brücken zwischen Menschen: Dankrede zur Verleihung des Friedrich-Gundolf-Preises 2004«, Jahrbuch der Deutschen Akademie für Sprache und Dichtung 2004, 56-64.
} 
erwartete Masing offensichtlich nicht, dass das deutsche Regime von Dauer sein werde. Eine Beschäftigung an der Universität unter der deutschen Besatzung war nicht allein mit dem moralischen Makel der Kollaboration, sondern zusätzlich auch geradezu mit einer Garantie nachheriger Sanktionierung belastet. Zum anderen erfüllte jedoch die wissenschaftliche Betätigung eine nicht unwichtige Funktion als Gegengewicht zu den überwältigenden Zeitläuften. Masing band sich an eine soziale und kulturelle Form der gelehrten Arbeit über philologische und theologische Fragen, die in der schwierigen ökonomischen Lage des unabhängigen Estland, danach unter dem institutionellen Druck der sowjetischen und deutschen Besatzungsmächte immer weniger Raum einnehmen konnte. Er fand sich in einer anachronistischen und marginalisierten Existenz wieder, die zunehmend nurmehr in der Arbeit selbst aufrechtzuerhalten war, da sie allen institutionellen und sozialen Beiwerks des üblichen akademischen Berufslebens entkleidet worden war.

Masing, der mehrere Dutzend Sprachen erlernt hatte, repräsentiert eine gelehrte Tradition linguistischer Hypertrophie, die in der Geschichte der Philologien insgesamt ebenso oft belächelt wie bewundert worden ist. Die Vielzahl seiner Projekte, die weltumspannende Ausdehnung seiner Interessen an semitischen, afrikanischen, austro- und melanesischen sowie amerikanischen Sprachen nahm so überhand, dass sie sich offenbar mit den Symptomen der psychischen Belastung verschränkte. Die Entgrenzung seiner Forschungen, ihre Ablösung von jeder Bindung an räumliche Gegebenheiten, stand in scharfem Kontrast zu seinen eigenen Bewegungen im physischen Raum, die ihn nicht weiter als bis Süddeutschland gebracht hatten.

In diesem Kontext war die linguistische Weltumspannung eine Art Pharmakon gegen die räumliche Beschränkung einerseits, die Gewaltdrohung des Weltkriegs andererseits. Die philologischen Überlegungen, die Masing 1942 erwähnt - nur ein kleiner Teil von über vierzig unabgeschlossenen Arbeitsvorhaben -, substantiierten sowohl das Leiden selbst als auch die Wirksamkeit des Gegenmittels. Wie das »Bromnatrium «, das er offenbar in erheblichen Mengen und ohne ärztliche Kontrolle einnahm, trug die Philologie zu den Symptomen bei, die sie zugleich linderte. Die lindernde Wirkung bestand darin, dass die wissenschaftliche Veräußerung die Imagination objektivierte; sie schuf eine Zone innerhalb der Wirklichkeit, die der überwältigenden Gefährdung durch die historischen Gegenwartsereignisse entzogen war. Die Aufrechterhaltung der philologischen Praxis erbrachte einen Nachweis der Verbundenheit des Gelehrten mit dem menschheitlich Besseren, das sich allerdings vom Schlechten der Menschheit auch nicht völlig ablösen ließ. Insofern hat man es hier tatsächlich mit einer jener »Gegenwelten« der Gelehrsamkeit zu tun, deren Bedeutung für Wissenschaftler insbesondere im Zusammenhang mit den Problemen des Exils nicht selten betont worden ist. ${ }^{17}$

Wegen dieser Bindung an das pharmazeutische Motiv der Gegenwelt als Gegenmittel besteht die Figur des Exils, wo sie als Metapher des intellektuellen Lebens dient, nicht unabhängig von der Frage nach der Zirkularität der Arzneimittelabhängigkeit und der Gewöhnung an Anästhetika. Masing brauchte eben 1942 schon vierzig Projekte, um die erwünschte Wirkung noch zu erzielen. Diese Wirkungsschwä-

17 Vgl. z. B. Kay Schiller, Gelehrte Gegenwelten: Über humanistische Leitbilder im 20. Jahrhundert, Frankfurt a.M. 2000. 
che der Philologie - der schriftlichen Wissenschaft vom Pharmakon der Schrift ${ }^{18}$ - bleibt als Herausforderung an das Verständnis der gelehrten Arbeit bestehen. In der Konfrontation zwischen den Vorgängen in der Welt und denen in der Gelehrsamkeit steht das Ergebnis jedenfalls von vornherein fest. Die Wissenschaft ist zur Gegenwehr nicht in der Lage; sie ist im Gegenteil zum Verzweifeln unterlegen. Masing stellt indirekt nicht nur die Hinfälligkeit des eigenen Lebens aus, wenn er (so schon 05.10.1940) die Ergebnisse seiner wissenschaftlichen Arbeiten an Littmann weiterreichen will, um sie vor dem eventuellen gewaltsamen Tod ihres Autors abzusichern. Er stellt auch die Schwäche und Verletzlichkeit der Gelehrsamkeit selbst zur Schau, ihre hilflose und sogar pathologische Gegenweltlichkeit, deren pharmazeutische Wirkung permanent im Schwinden begriffen ist und die deswegen noch ein zusätzliches Heilmittel erfordert, eben jenes Bromnatrium, mit dessen Hilfe allein die Zerfaserung der Arbeit an den quälend unerledigten Projekten erträglich wird.

Es handelt sich bei dieser Darstellung des Selbst in seinen pharmazeutischen Nöten auch um einen Widerspruch gegen die Konventionen der Rollenprosa des Professorentums, die gelehrte $»$ Persona $\ll,{ }^{19}$ wie man sie unter anderem auch an Littmann selbst studieren könnte. Masings Briefe geben ostentativ Auskunft über die Entstehung wissenschaftlicher Forschung als Ausdruck der Gefährdung von Leib und Leben des Forschers einerseits, von der Verletzlichkeit und, wenn man so will, Sterblichkeit auch der Forschung selbst andererseits. Das Verständnis dieser Eigenschaften ist keineswegs nur auf den Rahmen eines Kalküls bloßer Möglichkeiten beschränkt. Die Verletzlichkeit erweist sich vielmehr in der Realität der Verletzung, dem Wortsinn nach: im Trauma. Die Praxis der philologischen Arbeit stellt mithin ein Dokument der Verletzung her und legt Zeugnis davon ab, wie knapp der Weltphilologe der Auslöschung entkommen ist. Tatsächlich scheint für Masing zu gelten, dass der Weltbezug des Ausgeliefertseins nicht nur den Ursprung, sondern in gewisser Weise auch das Ziel der Philologie bilden soll. Damit verbunden ist eine implizite Abweisung aller konventionellen Annahmen über das movens der Forschung in der reinen Neugierde, in den Institutionen und gesellschaftlichen Verbänden, in der Methode oder im Charakter. Vom »Abschluss« des Menschlichen, von der Übersicht aus der Gipfelperspektive ist nirgends die Rede. Die Vorstellung des Exils - und sei es eines inneren - als einer Position epistemischer Privilegierung durch Distanz ist den Briefen Masings fremd. Seine Weltphilologie ist einer solchen Geste der Überlegenheit konstitutiv nicht fähig. Schon von daher verweigert sie sich der Vorstellung des historischen Endes. Doch ist diese Verweigerung nicht gleichbedeutend mit einer Abweisung aller geschichtsphilosophischen Überschreibungen. Im Gegenteil geht es Masing um eine andere, sogar gegensätzliche geschichtsphilosophische Position.

\footnotetext{
18 Dies mit Bezug auf Derridas Überlegungen zum Problem der platonischen Kritik der Schrift als einer Droge, die Gedächtnis und Vernunftgebrauch schädige, siehe »La Pharmacie de Platon« [1968], in: La Dissémination, Paris 1972, 77-214. Derrida weist der semantischen Mobilität von »pharmakon« zwischen Heilmittel und Gift eine zentrale Rolle zu.

19 Vgl. hierzu Lorraine Daston, Otto Sibum, »Introduction: Scientific Personae and their Histories«, Science in Context 16/1-2 (2003), 1-8; Herman Paul, »What Is a Scholarly Persona? Ten Theses on Virtues, Skills, and Desires«, History and Theory 53 (2014), 348-371.
} 


\section{IV.}

Auerbach etablierte 1952 eine geschichtsphilosophische Lesart der Welt der Weltphilologie vermittels der Figur des Endes, die er allerdings durch die zusätzliche Figur der wissenschaftlichen Re-Mythisierung zugleich einer tragischen Ironie unterwarf. Masing, wohl ohne Kenntnis der Arbeiten Auerbachs und unter höchst erschwerten Publikationsbedingungen, erarbeitete ein von dieser Lesart stark abweichendes Verständnis. 1960 fand er Gelegenheit, in der tschechoslowakischen theologischen Zeitschrift Communio Viatorum einen kurzen deutschsprachigen Text über seine Auffassung des Alten Testaments unterzubringen, in dem er zugleich seine eigene geschichtsphilosophische Position skizzierte. ${ }^{20}$ Die Deutung des Alten Testaments war für Masing zum Lebensthema geworden; er konnte kaum publizieren, hinterließ aber einen überwältigend umfangreichen Fundus unveröffentlichter Arbeiten zu dieser Thematik in estnischer Sprache. ${ }^{21}$

In der genannten Skizze setzt Masing zunächst sein Verständnis des Geschichtlichen auseinander. Zwei »Methoden« des Begreifens der Geschichte stünden demnach gegeneinander. Die erste sehe in der Geschichte nur verschiedene »Verkleidungen« immer derselben Idee. Diese Auffassung verweigere die Anerkennung eines über die eigene Mortalität gebildeten Geschichtsbegriffs (Heidegger steht hier als Gegner im Hintergrund) und halte den eigenen Tod und die Zeit letztlich für Illusionen. Dieses Geschichtsverständnis müsse allerdings anerkennen, dass es »niemals « die eine »Formel [wird] entdecken können, mit der Gott alle Koordinatensysteme seines Geistes gebildet hat und noch bilden wird «.22 Die zweite Auffassung dagegen halte die Zeit für wirklich und erkenne eine umfassende geschichtliche Bewegung an, daher notwendig eine fortschrittliche Entwicklung, denn »eine inhaltlose und ziellose Bewegung ist unvorstellbar, vielleicht ist sie auch logisch unmöglich« (ebd.). Hier dauere die Offenbarung Gottes noch an und vollende sich - erkennbar eine hegelianisierende Denkfigur - im Verlauf des historischen Prozesses. Die möglichen unterschiedlichen Auslegungen der Begriffe »Offenbarung « und »Welt« bedingten eine Bandbreite an Varianten dieses Geschichtsverständnisses. Allerdings führten alle möglichen Varianten notwendigerweise in dieselbe Antinomie: »Die Zeit kann nicht begrenzt sein, denn dann muss der Zeit eine Nicht-Zeit folgen, die undenkbar ist. Die Zeit kann nicht unbegrenzt sein, denn eine ewige Zeit ist keine Zeit mehr.« (ebd.) Aus diesem Grund hätten die Theologen den Begriff der Ewigkeit für die widersinnige Nicht-Zeit eingeführt; die Naturwissenschaft dagegen habe die Zeit universalisiert und damit allen Inhalts und aller Bewegung entkleidet. Nur die erste Geschichtsauffassung, die die Zeit selbst für einen Irrtum halte, lege den Begriff der Welt als Leben aus; die andere Auffassung, gleich auf welcher Seite ihrer internen Antinomie sie zu stehen komme, verstehe Welt ausschließlich als Tod.

\footnotetext{
20 Uku Masing, »Das Evangelium des Alten Testamentes«, Communio Viatorum 3/2 (1960), 123-132; vgl. auch ders., »Die Leute von Kumrān und das Alte Testament«, Communio Viatorum 3/3-4 (1960), 243-246.

21 Nõmmik (Anm. 1), 187.

22 Masing (Anm. 20), 124.
} 
In der Folge mokiert sich Masing über die schulische Fehlerziehung zum »Wiedererzählen«, zur Nacherzählung, in der aus einem gegebenen Text das angeblich »Wesentliche« wiederaufbereitet werde. In dieser Übung bestehe die Grundlage jener historischen Methode, aus der die entwicklungsgeschichtlichen Darstellungen der alttestamentlichen Wissenschaft des 19. und 20. Jahrhunderts entstanden seien. ${ }^{23}$ Diese Wissenschaft habe, da sie sich allein die Erarbeitung von dialektischen Entwicklungskontinuitäten aus der »Gesetzesreligion« des Judentums zum Christentum zur Aufgabe gesetzt habe, den Blick auf das Evangelium des Alten Testaments verbaut. Die wirkliche Botschaft der hebräischen Schriften liege in der Abweisung der Gesetzesreligion zugunsten der Prophetenreligion, von deren Unterdrückung einschließlich der wirklichen Ermordung und »Ausrottung « der Propheten - die Schriften indirekt oder direkt Zeugnis ablegten. Der Konflikt zwischen Prophetismus und Gesetzesherrschaft verweist aus Masings Sicht auf einen typologischen Grundkonflikt innerhalb der sich keineswegs »entwickelnden« Geschichte. Dieser Grundkonflikt findet zwischen zwei Typen vergesellschafteter Menschen statt, die Masing als »Raubritter« und »Schamanen« bezeichnet. Die Ersteren folgten einem Diktat der Gier des »Magens«, die Letzteren dagegen einem Gebot des »Geistes«. Diese Kategorien sind, wie unschwer zu erkennen, als Projektion aus einer recht einfach aufgefassten mittelalterlichen estnischen Geschichte angelegt, in der eine heidnische Urbevölkerung durch christliche Ritterorden gewaltsam kolonisiert und christianisiert wurde.

In der gleichursprünglichen Gegenüberstellung von gutem und bösem Prinzip lässt sich ein Echo gnostischer Positionen wiedererkennen. Zwar handelt es sich formal um ein Säkularisat, insofern die rein innerweltliche Struktur der Gegenüberstellung betont wird. Aber natürlich geht es eher um ein Spiel mit Denkmustern, die dem Theologen Masing tief vertraut waren. Ferner steht das Schema insgesamt auch im Zusammenhang mit Masings Auffassung von der intellektuellen Prägekraft der Grammatik (in deutlicher Affinität zur Sapir-Whorf-Hypothese). ${ }^{24}$ Den finno-ugrischen Sprachen zum Beispiel sollte nach Maßgabe der grammatikalischen Struktur ein Weltbegriff eignen, der sich etwa vom indoeuropäischen fundamental unterschied und der Auffassung von Zeit als Illusion deutlich näher stand. Doch sind im Sprachgebrauch Masings - er redet mehrfach von »Ausrottung « und »Abschlachtung « und betont die Tötbarkeit aller - die Erinnerungen an den Zweiten Weltkrieg wohl ebenfalls wirksam. Hinter der auch 1960 noch hypertrophen Theoriebildung (auch der Geist hat vielleicht einen Magen) steht neben anderem die persönliche Erfahrung des Ausgeliefertseins an das Böse in der Welt.

Dieses kurze und keineswegs ausreichende Referat soll vor allem darauf hinweisen, dass die Frage nach der Auslegung des Weltbegriffs für das Verständnis der Geschichte der Philologie unerlässlich bleibt; und dass in dieser Hinsicht historisch und begrifflich verschiedene, sogar widerstreitende Möglichkeiten bestanden und bestehen. Es scheint nicht einmal aus der Luft gegriffen, dass die geschichtstheologischen Signaturen, gleich ob mittelbar wie bei Auerbach oder unmittelbar wie bei Masing, einem Verhältnis interdependenter Differenzierung entsprangen, sich also

\footnotetext{
23 Masing (Anm. 20), $125 \mathrm{f}$.

24 Vgl. Nõmmik (Anm. 1).
} 
in ein Feld einschrieben mit Blick auf und gegen andere Positionen. Denn es ist denkbar, dass auch Auerbachs Formulierung eines Verständnisses von Weltphilologie schon einer Weigerung entsprach, sich auf eine Haltung in der Art der radikalen »Weltverachtung « ${ }^{25}$ des Masing'schen »Schamanismus « (in den Briefen erwähnt in selbstironischer Brechung, 14.05.1941) einzulassen. Das schlechte und böse Denken gehört eben gar nicht zu jenem Selbstausdruck der Menschheit, mit dem der Auerbach'sche Weltphilologe zu tun haben soll. Die »innere Geschichte « der Menschheit scheint eher eine des Guten, Wahren und Schönen zu sein. Diese versteckte Normativität, hinter der man die theologische Formierung des sogenannten Abendlandes gegen die Gnosis erkennen mag, kommt durch den Kontrast mit Masing immerhin deutlicher zum Ausdruck. Doch nährt die Verflochtenheit der geschichtstheologischen Signaturen mit Theorie und Praxis der Philologie einen Verdacht: dass nämlich die philologische Arbeit in ihrer weltlichen Zerstreuung unerlässliche Voraussetzung aller modernen theologischen Aufladungen des Denkens über Geschichte und Zeit ist. Keine Geschichtstheologie ohne Philologie, oder genauer: ohne die Welt(en) der Weltphilologie.

Warum ist aber in der Masing'schen geschichtstheologischen Signatur die Arbeit an den philologischen Details unerlässlich, und warum lohnt sich es daher auch, ihre Durcharbeitung nachzuvollziehen? Wohlgemerkt ist es beinahe kurios, dass für das Verständnis von Auerbachs Weltbegriff ein solcher Nachvollzug unnötig ist, obwohl der Autor von Mimesis ja besonders als Theoretiker des narrativen Details bekannt ist. Doch bei ihm kann die philologische Weltumspannung noch in die Zukunft aufgeschoben bleiben. Bei Masing hingegen muss sich die Möglichkeit der Weltphilologie in der Vergangenheit schon erwiesen haben; entweder weil man auf vorgängige Beispiele philologischer Praxis verweisen kann oder weil man eine Struktur innerhalb der historischen Wirklichkeit aufdecken kann, in der die typologischen Wiederholungen schon angelegt sind, die die Weltphilologie nachzeichnen muss. Letzteres ist offenbar Masings bevorzugter Beweismodus. Die Arbeitsvorhaben, von denen in den Briefen an Littmann die Rede ist, entsprechen nicht allein dem Muster einer Erforschung von Kulturtransfers, sondern suchen vielmehr Anschluss an die frühe strukturalistische Forschung, die in Estland seit der Zwischenkriegszeit rasch an Bedeutung gewonnen hatte. Hier ging es nicht mehr um Kausalität und Entwicklung, sondern eher um Form und Differenzierung. Prominentester Vertreter dieser Forschungsrichtung im Estland der Zwischenkriegszeit war der russische Exilant Walter Anderson (1885-1962), mit dem Masing befreundet war und dessen Einfluss in der folkloristischen Forschung z. B. auch bei Isidor Levin noch spürbar blieb. ${ }^{26}$ Anderson, der an der Universität Kasan die Revolutionszeit erlebt hatte und danach nach Estland geflohen war, nahm 1941 die Gelegenheit wahr, als »Volksdeutscher « nach Deutschland umzusiedeln.

\footnotetext{
25 Masing (Anm. 20), 131.

26 Vgl. zu Andersons wissenschaftlichem Projekt Michael Chesnutt, »The Great Crusader of Diffusionism: Walter Anderson and the Geographical-Historical Method «, Studies in Folklore and Popular Religion 1(1996), 11-26; Elo-Hanna Seljamaa, »Walter Anderson: A Scientist Beyond Historic and Geographic Borders«, in: Kristin Kuutma, Tiiu Jaago (Hrsg.), Studies in Estonian Folkloristics and Ethnology, Tartu 2005, 153-168.
} 
Es ist bemerkenswert, dass die in gnostischer Affinität ausgeführte geschichtsphilosophische Position Masings sich mit der strukturalistischen Forschungsrichtung verschränken konnte. Die Fragen, die ihn im Einzelnen beschäftigten, etwa nach der Gut-Böse-Markierung von Tagen im äthiopischen Kalender, nach der Wanderung von Marien- und Michaelslegenden, nach den weitläufigen Transfers einzelner Wörter, sollten die Einheit der Geschichte bestätigen, aber nicht als Entwicklungsgang, sondern als Struktur, als System formaler Variation. So öffnet der Blick über die Landschaft der philologischen Zerstreuung auch eine Perspektive auf die Art und Weise, wie der frühe Strukturalismus und das aus dem 19. Jahrhundert überkommene Spiel mit Theologemen des Geschichtsdenkens ineinandergreifen.

\section{V.}

Briefe von Uku Masing an Enno Littmann, (Nachlass Littmann, Staatsbibliothek Berlin, Sammlung Preußischer Kulturbesitz, NL 245, K. 20): Die folgende Wiedergabe der Briefe in chronologischer Reihenfolge erhält den originalen Textbestand, ohne Emendationen vorzunehmen, jedoch auch, ohne die zahlreichen Abweichungen von grammatikalischen und orthografischen Normen im Einzelnen kenntlich zu machen; nur bei besonders starken Abweichungen von der Norm wird die Texttreue mit (!) angezeigt. Alle Anmerkungen sind hinzugefügt.

1.

\section{Sehr geehrter Herr Professor!}

Sie hatten mir im vorigen Jahre erlaubt Ihnen zu schreiben; vielleicht hätte ich schon früher von dieser Erlaubnis Gebrauch machen sollen, aber Sie haben ja viel Interessanteres zu tun als meinen Brief zu lesen und deshalb habe ich nicht früher geschrieben. Obwohl ein später Dank, so möchte Sie (!) doch nochmals für Ihre Empfehlungen an Prof. Bertholet und Prof. Meissner ${ }^{27}$ danken, denn man hat mich sehr freundlich aufgenommen.

Ich habe von den semitischen Sprachen Äthiopisch, für meinen Spezialfach ausgewählt und habe einige Handschriften mir abgeschrieben, denn in Estland haben wir ja nichts, nur das was vor Dillmann ${ }^{28}$ erschienen ist, ist fast vollständig da und

\footnotetext{
27 Alfred Bertholet (1868-1951), Schweizer reformierter Theologe, Alttestamentler, Schüler Bernhard Duhms, seit 1928 Professor in Berlin; Bruno Meissner (1868-1947), seit 1921 Professor für Assyriologie und Semitistik in Berlin.

28 August Dillmann (1823-1894), Professor für alttestamentliche Theologie in Berlin, gilt als Wiederbegründer der philologischen Studien über altäthiopische Gegenstände in sprachwissenschaftlicher ebenso wie in epigrafischer und textphilologischer Hinsicht. Äthiopische Sprachstudien hatte er als Student der alttestamentlichen Theologie in Tübingen bei Heinrich Ewald treiben können. 1846-1848 hatte er die äthiopischen Sammlungen des British Museum und der Oxforder Bodleian Library katalogisiert: Catalogus codicum manuscriptorum orientalium qui in Museo Brittannico asservantur, 3: Codices Aethiopicos amplectens, London: British Museum, 1847; Catalogus codicorum manuscriptorum Bibliothecae Bodleianae oxoniensis, Pars VII: Codices Aethiopici, Oxford 1848. Littmann hatte noch kurz bei Dillmann studiert, wie er in seinen Fragment gebliebenen autobiografischen Aufzeichnungen erwähnt: »Leben und Arbeit«, hrsg. H. H. Biesterfeldt, Oriens 29/30 (1986), 1-101, hier: 14f. Die äthiopischen Studien entwickelten sich
} 
dann noch »Patrologia Orientalis «.$^{29}$ Eine Sache möchte ich gern herausgeben, näm-

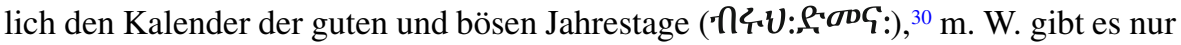
eine andere Handschrift in Paris und die habe ich bestellt, aber ob man sie hierher schickt, weiss ich nicht. Ich habe alle möglichen Kalender solcher Art verglichen (arabische, syrische, ägyptische) aber habe nichts Ähnliches gefunden. Es könnte

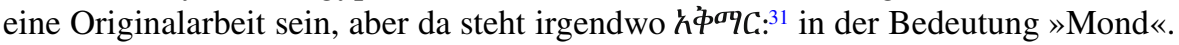
Doch fand ich unter 21 arabischen handschriftlichen Angaben nichts Ähnliches, denn die arabischen geben nur für die Monatstage etwas, oder Tabellen für das ganze Jahr. In dem äthiopischen ist aber ebenso wie in ägyptischen und assyrischen, für jeden Tag des Jahres etwas gesagt.

Der Text ist nicht immer übersetzbar, manchmal sehr vieldeutig und sonderbar und nach der einen Zeile die Conti Rossini aus der Pariser Handschrift abgedruckt hat, ${ }^{32}$ scheint diese ziemlich anders zu sein.

Wenn man mir die Erlaubnis zur Publikation gibt, so dachte ich in der »Acta et Commentationes « $^{33}$ unserer Universität zu veröffentlichen, aber sie haben keine äthiopischen Typen. Und ob in den deutschen Zeitschriften solche Sachen (und von Ausländern) angenommen werden, weiss ich nicht. Wahrscheinlich ist die ganze Arbeit nutzlos gemacht, mit mir geht es immer so. Sprachlich ist der Text ja nicht sehr wichtig, nur ein Paar neue Wörter die in Dillmann fehlen, wie $\boldsymbol{0}$ LC und HaDt, ${ }^{34}$ aber inhaltlich dürfte es doch der Veröffentlichung wert sein. Fraglich ist aber, ob man mir die Erlaubnis zur Veröffentlichung gibt.

insbesondere in Italien zur Kolonialwissenschaft. Als Nebenfach der Philologie der semitischen Sprachen bildeten sich jedoch auch in anderen Ländern äthiopistische Forschungstraditionen aus, ohne dass man einer der nationalen Schwerpunktbildungen Dominanz über die anderen zuschreiben könnte.

29 Seit 1904 erscheinende, besonders von französischen geistlichen Herausgebern gestützte Edition der ostkirchlichen Textüberlieferungen.

30 Translit. bəruh dəmäna (ich folge hier dem zu Littmanns Zeiten üblichen Umschriftsystem). Die Handschrift ist bei August Dillmann, Verzeichniss der abessinischen Handschriften, Berlin 1878 (Handschriften-Verzeichnisse der Königlichen Bibliothek zu Berlin, 3), 68, Nr. 75.3 verzeichnet (mit der Inhaltsangabe »Anweisung zur Tagewählerei «).

31 Translit. äqmar, Plural von $\mathbf{\phi a v}^{\circ} \mathrm{C}$, qämär, Mond. Wie schon August Dillmann, Lexicon Linguae Aethiopicae, cum indice latino, adiectum est vocabularium tigre dialecti septentrionalis, compilatum a Werner Munzinger, Leipzig 1865, 419 nachweist, handelt es sich um ein arabisches Lehnwort. Deswegen ist an Masings Bemerkung allenfalls die Pluralform überraschend.

32 Carlo Conti Rossini, Notice sur les manuscrits éthiopiens de la collection d'Abbadie, Paris 1917 (Auszug aus Journal Asiatique 1912-1915), Nr. 237, mit Übersetzung des Titels als »Nuage resplendissant«. Conti Rossini (1872-1949), einer der bedeutendsten italienischen Äthiopisten, beschäftigte sich auch später noch mit Problemen des Kalenders, siehe seine Tabelle comparative del calendario etiopico col calendario romano, Rom 1948.

33 Publikationsreihe der Universität Dorpat (Tartu), in der später Masings erste wissenschaftliche Monografien erschienen.

34 Translit. wärärä und zämätä. Gemeint ist Dillmann, Lexicon, wo die Formen in der Tat zu fehlen scheinen. Die zwei genannten Ausdrücke sind Verben, von denen wärärä im Amharischen nachgewiesen ist in den Bedeutungen (engl.) »invade, raid, pillage, plunder, swarm over (of ants), overrun, storm (of soldiers)《 (nach Wolf Leslau, Concise Amharic Dictionary, Wiesbaden 1976); und zämätä sowohl im Amharischen als auch im Ge'ez/Altäthiopischen in den Bedeutungen »go on a military expedition, plunder« (nach Wolf Leslau, Comparative Dictionary of Ge'ez, Wiesbaden 1987). 
Prof. Bulmerincq ${ }^{35}$ und ich haben Interesse für die Publikationen der Aksum Expedition $^{36}$ und Prof. Bulmerincq hat mich gebeten, daß ich mich an Sie mit der Bitte wenden sollte, ob Sie nicht vielleicht diese Veröffentlichungen für die orientalistischen Arbeiten aus der »Acta et Commentationes « unserer Universität austauschen wollen. Für die Anschaffung der alttestamentlichen und semitistischen Bücher bekommen wir nur etwa $250 \mathrm{Rmk}$ im Jahr, dadurch wird eine solche Bitte verständlich. Es gibt wohl nichts hoffnungsloseres auf der Welt als die Semitistik in Estland, obwohl Prof. Bulmerincq das nicht glaubt, aber die Sache bleibt doch so.

Und zum Schluss, wenn ich die Handschrift aus Paris und die Erlaubnis zur Veröffentlichung bekomme, darf ich dann mich an Sie wenden, wenn ich manchmal nichts anzufangen weiss? Und vielleicht wissen Sie eine Zeitschrift, die solche Sachen annimmt?

Berlin W 15, Fasanenstr. 61

Mit bestem Gruss

1. VI. 1933

Ihr ergebenster + Hugo Masing $+^{37}$

\section{2.}

\section{Sehr geehrter Herr Professor,}

ich hoffe, dass ich Ihnen zu Ihrem fünfundsechzigsten Geburtstage meine Doktorarbeit mit einem Grusse senden kann. ${ }^{38}$ Jedoch dauert es noch eine Woche bis die Arbeit fertig gedruckt ist und da es keinen Lehrstuhl gibt bei dem ich jetzt die Arbeit verteidigen konnte, wird sie keine Doktorarbeit sein. Die theologische Fakultät wurde ja liquidiert und die Universität hat keinen Gebrauch für meine semitisti-

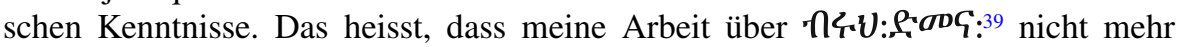
erscheinen wird und ich nicht mehr weiter arbeiten kann. Augenblicklich tröste ich mich mit der Tatsache, dass ich bei Ihnen doch etwas gelernt habe wie auch meine Ausführungen über die äthiopische Übersetzung des Obadja hoffentlich zeigen werden.

Ich wünsche Ihnen, dass Sie noch viele Jahrzehnten ruhig und fruchtbar arbeiten könnten und noch viel Neues entdecken würden.

\footnotetext{
35 Alexander von Bulmerincq (1868-1938), Theologe und Orientalist, Professor an der Universität Tartu.

36 Gemeint ist die Publikation der Forschungsergebnisse der von Littmann im Jahr 1906 geleiteten Expedition zu den altäthiopischen Altertümern in Aksum, Enno Littmann, Theodor von Lüpke, Daniel Krencker, Deutsche Aksum-Expedition, 4 Bde. hrsg. von der Generalverwaltung der Königlichen Museen, Berlin 1913. Siehe auch Steffen Wenig u. a. (Hrsg.), In Kaiserlichem Auftrag: Die Deutsche Aksum-Expedition 1906 unter Enno Littmann, 1: Die Akteure und die wissenschaftlichen Unternehmungen der DAE in Eritrea, Aichwald 2006; 2: Altertumskundliche Untersuchungen der DAE in Tigray/Äthiopien, Wiesbaden 2011.

37 Masing, der aus einer Familie deutschbaltischer Herrnhuter stammte, war auf den Namen »Hugo« getauft worden und bediente sich meist der Estnisierung »Uku«.

38 Littmanns Geburtstag war am 15.9. Masings Doktorarbeit ist die Schrift über den Propheten Obadja, deren erster Teil in den Acta et Commentationes im Jahr 1937 erschienen war, deren zweiter Teil jedoch ungedruckt blieb: Der Prophet Obadja, I: Einleitung in das Buch des Propheten Obadja. Acta et Commentationes, B: Humaniora, 41, Tartu 1937.
}

39 S.o. Anm. 30. 
J. Hurda 9-3, Tartu

Hochachtungsvoll

URSS (Estonie)

Ihr

13. IX 1940

+Uku Masing+

3.

Sehr geehrter Herr Professor,

dass ich so etwas wie ein Lügner vor Ihnen bin, ist nicht meine Schuld. Es waren von meiner Arbeit nur etwa dreissig Seiten noch zu drucken, die Korrekturen waren gelesen und alles sonst in Ordnung als es bekannt wurde, dass nicht weitergedruckt wird. Meine Arbeit sieht wohl nicht wie eine theologische aus, aber immerhin ich habe über einen Propheten geschrieben. Wie es scheint gibt es keinen Gebrauch für mich in der philosophischen Fakultät, für Prof. L. Gulkowitsch ${ }^{40}$ (früher in Leipzig) vielleicht doch.

Ich bitte also mich zu entschuldigen, dass ich mein Versprechen auch jetzt noch nicht erfüllen kann. Damit meine »Entdeckung « nicht verloren geht sage ich es Ihnen. Ich habe gefunden, dass es eine »hebräische Rezension« der äthiopischen Bibelübersetzung nicht gibt, die Stellen, die dafür zu sprechen scheinen, sind von den katholischen Kommentaren, des XVI. und XVII. Jahrhunderts abhängig. Ausserdem habe ich gefunden, dass die Loefgrensche Gruppierung der Handschriften (bei Daniel) ${ }^{41}$ wenigstens für Obadja nicht gültig ist und dass auch die äthiopischen Bibelkommentaren ziemlich stark europäisch beeinflusst sind. Sonst wäre vielleicht noch eine Kleinigkeit wichtig, die vielleicht sie (!) interessieren wird. ספרד in Ob. $20^{42}$ worüber so viel geschrieben ist, ist wie die von Ihnen herausgegebene lydische Inschrift (Grabstele des Mane [Mani?] des Sohnes Kulmli) zeigt sicher Sardes. ${ }^{43}$ Wahrscheinlich wird meine Arbeit eingestampft oder als Makulatur verwendet, Sie können also meine »Entdeckungen « wenn Sie es wollen, selbst benutzen oder Ihren Schülern weitergeben - wenn sie etwas Wert haben. Hier gibt es jedenfalls ausser mich keinen, der entscheiden könnte, ob sie irgendwie wichtig sind. Wenn Sie et-

\footnotetext{
${ }^{40}$ Lazar Gulkowitsch (*1898) war als Flüchtling vor der sowjetischen Revolution zunächst nach Königsberg, dann nach Leipzig gegangen, dort 1925 habilitiert worden und als Privatdozent lehrberechtigt gewesen. 1932 wurde er an der Philosophischen Fakultät zum Extraordinarius für Wissenschaft des Judentums ernannt, aber 1933 wurden ihm Anstellung und Venia entzogen, und er emigrierte nach Tartu. Er und seine Familie wurden im Sommer 1941 - ein genaues Datum ist nicht feststellbar - von den Deutschen ermordet. Gulkowitsch ist ein weitgehend vergessener Pionier der begriffsgeschichtlichen Forschung. Vgl. Siegfried Hoyer, »Lazar Gulkowitsch an den Universitäten Leipzig und Dorpat (Tartu)«, in: Judaica Lipsiensia: Zur Geschichte der Juden in Leipzig, hrsg. von der Ephraim Carlebach Stiftung, Leipzig 1994; Urmas Nõmmik, »Lazar Gulkowitsch und das Seminar für jüdische Wissenschaft an der Universität Tartu (Dorpat)«, 2 Teile, Judaica 61/4 (2005), 351-372 und 62/1 (2006), 1-42.

41 Oscar Löfgren, Die äthiopische Übersetzung des Propheten Daniel. Nach Handschriften in Berlin, Cambridge, Frankfurt am Main, London, Oxford, Paris und Wien, zum ersten Male herausgegeben und mit Einleitung und Kommentaren versehen, Paris 1927.

42 Translit. s-p-r-d, übliche Übersetzungen sind »Sefarad« (Luther-Bibel), »Sarepta« (Vulgata) und »Zarephath« (King James Version). Der lydische Name für Sardis war »Sfard «.

43 Hierbei handelt es sich um eine 1912 entdeckte lydisch-aramäische Bilinguis in Sardis, Enno Littmann, Sardis: Publications of the American Society for the Excavation of Sardis, vol. IV Lydian Inscriptions, Part I, Leiden 1916, 23-40.
} 
was Näheres über sie erfahren wollen bin ich bereit zu schreiben obwohl ich bald vielleicht kein Geld mehr für Briefmarken haben werde.

J. Hurda 9-3, Tartu

URSS (Estonie)

Hochachtungsvoll +Uku Masing+

5. X 1940

4.

Sehr geehrter Herr Professor,

dass ich von Ihnen wieder einen Brief empfangen habe, hat mir eine grosse Freude bereitet, ebenso, dass mein nichteingeschriebener Brief doch in Tübingen angekommen ist. Ich wagte gar nicht darauf zu hoffen. Dass an dieser »Arbeit« etwas zu verbessern wäre, weiss ich nicht, hinzuzufügen wohl, denn der Mensch lernt solange er lebt. Da könnte noch ein Absatz über die Sage über den Nibelungenschatz, der noch a.c. gehoben werden soll, hinzugefügt werden. Vielleicht auch etwas über das Reich Tsin zwischen 240-220 a. Ch., aber ich glaube, dass ich auch darüber etwas gesagt habe.

Haben Sie vielen Dank für die Angaben über »1001 Nacht«. Ich hoffe, dass ich doch Ihnen die Bulaqer Ausgabe ${ }^{44}$ mit vielem Dank einmal zurücksenden kann, wenn nicht ich, so irgendjemand, die oder den ich noch beauftragen kann. Da meine estnische Übersetzung nicht länger und nicht kürzer sein darf als die russische Übersetzung, muss ich manchmal doch die Calcuttaer Ausgabe vergleichen. Viele Geschichten sind, so viel ich aus Ihrer Übersetzung verglichen mit der Bulaqer Ausgabe sehe, wörtlich gleichlautend. Es wäre vielleicht eine interessante Arbeit die Abweichungen einmal systematisch durchzunehmen. In Leningrad gibt es eine Calcuttaer Ausgabe, die kann ich wahrscheinlich vergleichen, denn sowieso muss ich die Übersetzung dreimal schreiben. Es ist ja die erste Übersetzung aus dem Arabischen ins Estnische und ich habe noch keine Gewöhnung, Reimprosa versuche ich wiederzugeben, obwohl der Sinn darunter sehr zu leiden hat viel mehr als in der deutschen Sprache, Gedichte natürlich auch, denn es gibt noch weniger Reime und den Rhythmus herauszubringen ist unmöglich. ${ }^{45}$ In der russischen Übersetzung sind die Gedichte rhythmisch übersetzt aber ungereimt. Es gibt natürlich Ausdrücke, die ich aus den Wörterbüchern nicht finde und die grösste Mühe machen mir die ف's und ف's. ${ }^{46}$ Ich bin ganz ängstlich Ihnen gegenüber. Aber ich habe auch jetzt nicht,

\footnotetext{
44 Die komplexe Textgeschichte der »1001 Nacht« zugrunde liegenden Handschriften ist vielfach diskutiert worden, siehe zuletzt Paulo Lemos Horta, Marvellous Thieves: Secret Authors of the Arabian Nights, Cambridge, MA 2017. Littmann hatte sich für seine eigene Übersetzung auf die Kollation verschiedener arabischer Ausgaben verlassen, die aber ihrerseits fast alle unter dem Erfordernis der Anpassung an die europäische Tradition der sehr freien, andere Stoffkreise (Sindbad) beimischenden und überdies stark textentstellenden Märchensammlung in der Nachfolge Antoine Gallands entstanden waren. Masing arbeitete in Kenntnis von Littmanns Übersetzung und hatte zu diesem Zeitpunkt offenbar vor, die Bulaqer (= Kairoer) und Kalkuttaer Ausgaben zu kollationieren.

${ }^{45}$ Littmanns Ausgabe gibt die Gedichte in Metrum und Reim wieder, Die Erzählungen aus den tausendundein Nächten: Zum ersten Mal nach dem arabischen Urtext der Calcuttaer Ausgabe vom Jahre 1839, 6 Bde., Leipzig 1921-1928.

${ }^{46}$ Gemeint sind wohl die Buchstaben $q \bar{a} f$ und $f \bar{a}$ ' des arabischen Alphabets, wobei über der zweiten Form allerdings die Punktierung fehlt, sodass sie einem 9 , wāw ähnelt.
} 
wo doch der Kontrakt mit dem Verlage untergeschrieben ist, keinen Glauben an die Arbeit. ${ }^{47}$ Ich werde etwa 3900 Rubel für die Übersetzung etwa eines Viertels des Textes bekommen und zwar wird für jeden abgelieferten Teil gezahlt. Vielleicht am Ende dieses Monats werde ich ein Zehntel abgeben. Vielleicht werde ich dann auch einen stärkeren Glauben an den Fortgang der Arbeit bekommen.

Ich bin nicht sehr glücklich bei der Arbeit, wahrscheinlich sind die Nerven am Rande des Nichtseins als Folge der Krankheit oder bin ich auch erblich belastet, unter den Verwandten gibt es Epileptiker. Jedenfalls ist jeder Tag für mich unruhiger als der vergangene und ich lebe hauptsächlich von Bromnatrium, das sehr billig ist (etwa 5 Kopeken fürs Gramm) und von Cofeopyrin. ${ }^{48}$ Wie lange es so geht, ahne ich nicht. Für »ein bisschen anormal« wurde ich ja immer gehalten, nun hätte man Recht dazu. Ich habe Ihnen schon einen Brief geschrieben, aber der war zu īnāartig. ${ }^{49} \mathrm{Ob}$ dieser besser ist, weiss ich nicht. Wenn ich kein Bromnatrium hätte würde ich immerdar an meine »Entdeckungen « denken, ohne eine einzige wahrlich ausführen zu können. Etwa vierzig Arbeiten müsste ich beendigen, aber ich weiss nicht mehr, welche. Ohne Bromnatrium denke ich immer an die, die ich nicht um mich herumliegen sehe, mit diesem existieren sie für mich nicht mehr. Deshalb danke ich Ihnen von Herzen für die Adresse des Herrn Major a. D. Dr. phil. G. Friederici ${ }^{50}$ Ich kenne einige seiner Arbeiten, ein paar kleinere besitze ich sonderbarerweise und hoffe, dass wenn nicht er, so irgendeiner seiner Bekannten sich vielleicht für diese Dinge interessieren wird. - Ausserdem kann es möglich sein, dass ich bald ausziehen muss, denn mein »Vertrauensmann« (einer, der eine gewisse Anzahl von Häusern zu überwachen hat und die Zettel für die Miete ausfüllen muss) braucht eine Wohnung und sucht nun nach Gründen um von mich loszuwerden.

Mein lieber Freund, Prof. Dr. L. Gulkowitsch soll in Leningrad Professor und Akademiker werden, wenigstens spricht er selbst davon. Ob er mich in Ruhe lässt, weiss ich nicht. Er ist mir böse, weil einer von seinen Schülern zu mir, zu diesem »Wirrkopf « übergekommen ist. Vielleicht hat er auch andere Gründe, einmal berührte ihn die nicht zu verändernde Tatsache sehr unangenehm, dass ich Sie kenne, er wollte der einzige sein, der in so einem Hinterlande berühmte Leute kennt, dann hat er wie es scheint auch daran gehofft, dass er der Nachfolger von Professor von Bulmerincq werden soll. ${ }^{51}$ Einen Ruf nach Jerusalem hat er nicht angenommen, weil

\footnotetext{
47 Neu herausgegeben als Tuhande ja ühe öö jutte. Araabia keelest tõlkinud Uku Masing, Tartu 2007.

48 Natriumbromid, heute nicht mehr gebräuchliches Sedativ und Antidepressivum, das die Symptome, die Masing damit bekämpfen wollte, auch auslösen kann (im Zusammenhang mit dem als »Bromismus « bekannten Vergiftungssyndrom). »Cofeopyrin« scheint unter diesem Namen nicht nachweisbar zu sein; es handelt sich wohl um ein mit Coffein als Wirkverstärker versehenes Acetylsalicylsäurepräparat (weil dem Namen nach ein Antipyretikum); das Preisverzeichnis deutscher pharmazeutischer Spezialpräparate: Rote Liste 1939, Berlin 1939, 154 weist mit dieser Zusammensetzung die Produkte »Coffetylin« und »Coffex (früher Coffeocitrin)« aus.

49 Qina $\bar{a}$, hebr. Totenklage.

50 Georg Friederici (1866-1947), der als Offizier im Ruhestand umfangreiche kulturanthropologische und linguistische Forschungen über polynesische und nordamerikanische Themen betrieb.

51 Bulmerincq ging in einem Empfehlungsschreiben an Littmann 8. April 1932, ebenso in einem weiteren Brief 18. April 1934, NL Littmann, K. 5, davon aus, dass Masing sein »präsumptiver Nachfolger« sei, da er ein entsprechendes Stipendium erhalten habe.
} 
man seine Bedingungen (Dekan der Fakultät und Vollmächte zu Reorganisierung) abgeschlagen hat. Wenn er doch einmal nach Leningrad gehen würde!

Nochmals vielen Dank für Ihren Brief. Ich denke jetzt sehr oft an die Zeit vor neun Jahren, als die Freude an Arbeit und die Arbeitsmöglichkeiten so gut waren. Was ich in Tübingen und Berlin gelernt habe, macht vielleicht drei Viertel davon aus, was ich überhaupt weiss. Damals brauchte ich an gar nichts Andres zu denken als nur an Lesen, brauchte nichts zu tun, was ich nicht wollte, nicht an einen Beruf und an das Verdienen zu denken. Manchmal fühle ich, dass die Zeit als ich Ihre Vorlesungen hörte, näher ist als alles was nachher geschehen ist. Und ausserdem war Süddeutschland für so einen Nordländer wie ich und für so einen dummen ästhetisch veranlagten Kerl auch ein schönes Land. Ich hoffte einmal nach Marquesasinseln zu gehen, nun bin ich sehr glücklich, dass ich doch etwas gesehen habe und irgendwo gewesen bin wo es auch noch im Oktober warm ist. Diejenige, die behaupten, dass der Schamanismus eine arktische Erscheinung ist, haben sicher Recht, die ewige Kälte macht die Menschen ängstlich und furchtsam. Aber ich beginne wieder eine īnā zu singen, das verstehe ich noch ziemlich gut. Wenn das der letzte Brief sein sollte bei dem ich noch ziemlich verständig bin, so muss ich noch sagen, dass ich Ihnen sehr dankbar bin, dass Sie da sind. Vielleicht sind die Magnolien, die Herbstzeitlosen auf dem Österberge und die preussische Staatsbibliothek viel unwichtiger gewesen. Irgendjemand weiss das, ich wage nichts zu sagen. Für einen Esten ist nicht unmöglich, dass er auch mit dreissig Jahren weinen kann, vielleicht nicht so oft wie die Araber in $» 1001$ Nacht«, aber mehr in sich hinein.

14.V 1941

Möge es Ihnen sehr gut gehen +Uku Masing+

\section{5.}

Sehr geehrter Herr Professor,

ich benutze die erste Möglichkeit Ihnen zu schreiben, eine Dame aus Berlin war so freundlich und versprach mir einen Brief an Sie mitzunehmen. Ich hätte viel zu schreiben, aber Vieles wissen Sie wahrscheinlich aus Zeitungen und damit Sie zu belästigen will ich nicht. Wie man hier sagt, volle sechs Wochen war auch ich »im Wald «. Gerade langweilig war es eben nicht, aber Angst vor der Ergreifung oder Mobilisierung zu haben, war an sich wieder keine fröhliche Angelegenheit. An sich ist es sonderbar, dass ich übrig geblieben bin, da sehr viele Menschen fehlen, die man wahrscheinlich oder sicher nie mehr wiedersehen wird.

Aus der theologischen Fakultät bin ich gestern herausgetreten und bin also bald kein Professor mehr. Ich wollte es schon »vor den Russen« tun, aber damals wären meine Gründe nicht genügend gewesen. Die moralische Art, die ich leider habe, passt nicht zu einem solchen Amt. Augenblicklich weiss ich noch nicht, was ich tun werde um leben zu können, aber irgendwelche Arbeit wird meine Frau oder werde ich doch finden.

Von meiner Doktorarbeit über Obadja habe ich einen Exemplar retten können, die übrigen haben die Kommunisten als Makulatur behandelt. Eine öffentliche Verteidigung, die zum Erhalten des Doktorgrades der Theologie notwendig gewesen

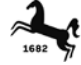


wäre, wird kaum zu Stande kommen. Ich habe keine Opponenten mehr und der, der noch da ist, will es nicht tun. ${ }^{52}$ Es wird wahrscheinlich Alles so bleiben wie es ist.

Ihre Bulāqer Ausgabe würde ich Ihnen gern zurücksenden, denn es ist nicht zu hoffen, dass die Übersetzungsarbeit noch weiterhin möglich ist. Bisher habe ich leider noch keine Möglichkeit dazu gefunden, aber einmal muss es doch eine geben.

Augenblicklich habe ich Arbeit genug, die jüdischen und hebräischen Bücher bleiben meistens für die hiesige Universität, ich katalogisiere sie - als eine Gegengabe an die Universität, die bisher für meine Notwendigkeiten Sorge getragen hat. Es würde eigentlich vielleicht ein Jahr dauern bis ich damit fertig werde, aber es scheint so, dass nicht ich die Arbeit beendigen werde.

Ich wäre sehr glücklich wenn ich von Ihnen wieder einen Brief haben könnte. Hoffentlich geht es Ihnen gut - von der hiesigen Perspektiven aus bin ich schon gewöhnt zu denken, dass es kaum Menschen gibt denen es gut geht. Vielleicht verlerne ich das bald, aber bisher bin ich nicht so weit gekommen.

Manchmal denke ich an die orientalischen Handschriften in Russland, welche und wie viele davon übrig bleiben werden. Früher habe ich an die Ausgaben der $»$ Wunder Michaels« und der »Wunder Marias $\ll^{53}$ gedacht, meine Frau ist einmal Setzerin in einer Druckerei gewesen, etwas äthiopisch kann sie und Typen hat eine hiesige Druckerei auch etwas, sodass wir zusammen ein Paar Bücher hätten machen können. Aber irgendwie muss das eine nichtgute Träumerei gewesen sein.

Wenn Herr v. Bulmerincq ${ }^{54}$ da ist, seien Sie so gut und grüssen Sie ihn von mir. Ihnen selbst wünscht alles Gute und fröhliche Weihnachten

J. Hurda 9-3

9.XII 1941

Ihr ergebener + Uku Masing+

\section{6.}

\section{Sehr geehrter Herr Professor,}

Vielen Dank für Ihren Brief, den ich vor ein Paar Tagen erhalten habe und für die Anteilnahme an dem Schicksal meiner Arbeit. Nun hoffe ich, dass Sie im Laufe der Zeit auch schon meinen Brief von Mitte Februar erhalten haben. Darin habe ich geschrieben, dass aus der Verteidigung der Arbeit doch nichts geworden ist und heutzutage ist die Lage genau dieselbe. Es geht uns nicht schlecht, meine Frau verdient etwa 90 Mark monatlich und ich habe fast den ganzen Tag frei zum

\footnotetext{
52 Gemeint ist hier das im russischen Universitätssystem übliche, altertümliche Verfahren einer Disputation mit einem Opponenten.

53 In beiden Fällen liegen komplexe Überlieferungstraditionen mit multiplen Manuskripten vor; zu den Wundern Mariae s.u., zu den Wundern des Erzengels Michael, die in der Korrespondenz nicht weiter erwähnt werden, ist allenfalls zu bemerken, dass die Entwicklung einer entsprechenden Texttradition zu den Eigenheiten der äthiopischen Kirchen- und Königsgeschichte insbesondere seit dem 15. Jahrhundert gehört.

54 Gemeint ist hier Alexander von Bulmerincq jr. (1909-1945), genannt Axel, der Sohn des 1938 verstorbenen Professors. Bulmerincq jr. hatte ebenso wie Masing bei Littmann studiert, war danach in Deutschland geblieben und fungierte während des Krieges als Dolmetscher, unter anderem im Afrikakorps der Wehrmacht. Er wurde im Februar 1945 bei Kampfhandlungen in der Nähe von Posen getötet.
} 
Arbeiten. Sehr glücklich bin ich dabei nicht, aber schliesslich gewöhnt man sich an Allem.

Ich arbeite jetzt an einer Grammatik der Quichésprache ${ }^{55}$ und hoffe dass es etwas klarer und gründlicher wird als die bisherigen. Daneben auch etwas Äthiopisch aber damit was ich selbst besitze und was ich noch haben kann ist nicht viel anzufangen.

Sehr dankbar wäre ich Ihnen wenn Sie Herrn v. Bulmerincq von mir viel Glück in seiner Arbeit wünschen würden.

Hoffentlich geht es Ihnen immer gut. Auch bei uns ist der Frühling schon da, der so lange erwartete. Über sechs Monate lang hat ja diesmal der Winter gedauert.

J. Hurda 9-3, Tartu

Mit besten Grüssen und Wünschen

16.IV 1942

Ihr ergebener

+ Uku Masing+

\section{7.}

Sehr geehrter Herr Professor,

vielen Dank für Ihren Brief und für den Mut, den Sie mir dadurch gegeben haben. Leider habe ich noch nichts Fertiges und über die abessinischen Monatsnamen vielleicht auch nie. Es gibt hier sehr wenig afrikanistische Literatur und ich müsste wenigstens die meisten Kalender der Nachbarvölker der Abessinier kennen um irgendwie klarer zu sehen. Ich weiss aber zu wenig. Mit den »Wunder Mariae« geht es etwas besser, aber ich kann nur Fragen formulieren, habe vielleicht auch viel unnütze Arbeit getan. ${ }^{56}$ Es war meine Absicht zuerst alle Legenden, die in den äthiopischen Handschriften vorhanden sind zu katalogisieren, aber ich konnte nur Zotenberg, ${ }^{57}$ Conti Rossini, Rhodokanakis ${ }^{58}$ und Grohmann ${ }^{59}$ haben, Wright ${ }^{60}$ hat ja keine Inhaltsangaben und von den Handschriften in Berlin kenne ich nur eine. Ich weiss, dass es noch Kataloge gibt, die hier vorhanden sind (Zettersteen, Turaev), ${ }^{61}$ aber meine Bewegungsmöglichkeiten sind sehr begrenzt. Dann habe ich

\footnotetext{
55 D.i. Quechua.

56 In der Tat stand der italienische Äthiopist Enrico Cerulli im Begriff, zu dieser Texttradition eine einschlägige Studie zu publizieren, Il Libro etiopico dei miracoli di Maria e le sue fonti nelle letterature del Medio Evo latino, Rom 1943.

57 Hermann Zotenberg, Catalogue des manuscrits éthiopiens (Gheez et Amharique) de la Bibliothèque Nationale, Paris 1877.

58 Nikolaus Rhodokanakis, Die äthiopischen Handschriften der K.K. Hofbibliothek zu Wien, Wien 1906.

59 Adolf Grohmann, Äthiopische Marienhymnen, Leipzig 1919.

60 William Wright, Catalogue of the Ethiopic Manuscripts in the British Museum, Acquired since the Year 1847, London 1877. Diese Arbeit ergänzte Dillmanns Katalog von 1847, da sich die Sammlung nach dem britischen Feldzug gegen Kaiser Tewodros (Theodoros) II. 1867-68 durch geplünderte Manuskripte bedeutend erweitert hatte. Tatsächlich hatte Tewodros selbst, wie Wright einleitend (iii-iv) ausführt, eine landesweite Sammlung von Manuskripten veranlasst, die in seiner Festung Magdala aufbewahrt wurden und nach deren Eroberung den Briten in die Hände fielen. Siehe hierzu Volker Matthies, Unternehmen Magdala: Strafexpedition in Äthiopien, Berlin 2010.

61 Karl Vilhelm Zetterstéen, Die arabischen, persischen und türkischen Handschriften der Universitätsbibliothek zu Uppsala, 2 Bde., Uppsala 1930-1935; und wohl Boris Turaev, Monumenta Aethiopiae Hagiologica, 3 Bde., Leipzig 1902-1905.
} 
Mussafias »Studien zu den mittelalterlichen Marienlegenden $\ll^{62}$ vorgenommen, bin aber noch nicht fertig mit denen, dann werde ich auch Poncelet's Arbeit in Analecta Bollandiana $^{63}$ durchnehmen und hoffe, dass es mir gelingt eine Quelle aufzufinden, die diese 33 Legenden bietet, die Conti Rossini für den Grundstock hält. Eigentlich bin ich nicht ganz sicher ob er völlig im Rechten ist, denn Wright LXXVI soll ja zwischen 1434 und 1468 geschrieben worden sein enthält aber schon 51 Legenden. Ist es vorstellbar, dass so bald nach der Übersetzung, höchstens zwanzig Jahre, die Sammlung schon auf fast das Doppelte gestiegen ist? Vielleicht gab es doch verschiedene Übersetzungen, denn es gibt ja Handschriften, die von diesen 33 nur einige, und auch diese zerstreut haben. Dann kann ich auch von einem Gefühle nicht loswerden: war die Grundlage nicht eine Sammlung hergestellt für das Königreich Jerusalem? Lateinisch ist die Vorlage sicher gewesen, denn der Judenknabe in ח.C. ${ }^{6}{ }^{6}{ }^{64}$ muss derjenige in »civitate Bituricensi« ${ }^{65}$ sein, arabisiert vielleicht Wenn Budge ${ }^{67}$ Recht hat, dass Wright LXXVII noch älter ist als LXXVI, ${ }^{68}$ wird die Lage noch dunkler, denn die enthält ja 160 Legenden und soweit sie in der Übersetzung vorhanden sind, hat jede eine europäische Parallele. Ich beginne jedenfalls den Glauben an den Grundstock zu verlieren. Wenn aber diese 22 erst in Abessinien »kanonisiert« worden sind, ist es vielleicht unmöglich die Handschrift aufzufinden, die der Quelle am nächsten läge. Hoffentlich werde ich noch etwas klüger, aber die Handschriften in Wright sind eine Menge von Steinen im Wege.

Ich habe ziemlich viel Zeit zum Arbeiten, aber die Gesundheit ist nicht die beste und es mangelt an Büchern, noch mehr an Zeitschriften. Einige Freunde versorgen mich mit Büchern aus der Universitätsbibliothek, wo aber neuere Werke fast voll-

\footnotetext{
62 Adolf Mussafia, Studien zu den mittelalterlichen Marienlegenden, 5 Bde. Wien 1887-1898. Diese textkritische Diskussion der Beziehungen zwischen den mittelalterlichen lateinischen Handschriftensammlungen war grundlegend für spätere stoffgeschichtliche Untersuchungen.
}

63 Wohl Albert Poncelet, »Miraculorum B. V. Mariae quae saec. VI-XV latine conscripta sunt: Index«, Analecta Bollandiana 21 (1902), 241-360.

64 Translit. äth. T'iros.

65 D.i. Bourges; gemeint ist eine lateinische Legende, nämlich die von Eugen Wolter, Der Judenknabe: 5 griechische, 14 lateinische, und 8 französische Texte, Halle 1879 behandelte vom »Judenknaben im Ofen«, der mit seinen christlichen Spielgenossen an der Kommunion teilgenommen hatte, vom Vater deswegen in den Ofen geworfen, daraus aber von der Jungfrau Maria errettet wurde. Die Handlung wird seit dem 12. Jahrhundert in Bourges angesiedelt, aber die Legende geht in der lateinischen Literatur bis auf Gregor von Tours zurück und hat noch ältere griechische Vorläufer. Der Stoff der Marienlegenden ist vielfach anti-jüdisch; und wie Mussafia (Anm. 62) noch deutlicher zeigt, ist die Legende vom Judenknaben im Ofen Bestandteil fast aller entsprechenden Sammlungen, einschließlich der äthiopischen, wie auch Wolter (Anm. 65), 20, bereits unter Verweis auf Zotenbergs Katalog erkannt hatte.

66 Translit. $b$-trūs

67 Gemeint ist der Ägyptologe und Orientalist E. A. Wallis Budge (1857-1934), der auch mehrfach zu den äthiopischen Marienlegenden publiziert hatte, vgl. Legends of Our Lady Mary the Perpetual Virgin and her Mother Hannâ, translated from the Ethiopic manuscripts collected by King Theodore at Makdala and now in the British Museum, London 1922; ders., One Hundred and Ten Miracles of our Lady Mary, translated from Ethiopic manuscripts, London 1933.

68 Bei diesen beiden Handschriften handelt es sich um Sammlungen von Marienlegenden; Nr. LXXVI enthält den Hinweis, sie sei auf Veranlassung des Kaisers Zar'a Ya'qob (1434-1468) aufgeschrieben worden. Nr. LXXVII enthält keine entsprechende Angabe, wird aber von Wright ebenfalls auf das 15. Jahrhundert datiert. 
ständig fehlen. Ich hoffe, dass es Ihnen nicht sehr langweilig wird, wenn ich Ihnen noch von einer Sache spreche, die ich jetzt vielleicht gelöst habe. Es gibt nämlich in den mordwinischen, den ostseefinnischen und lappischen Sprachen ein Wort *makou $>$ Preis usw. $<$ Ich habe schon lange gedacht, dass das Wort aus dem akkadischen miksu

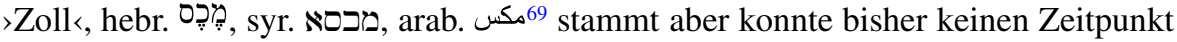
für die Entlehnung finden, denn da das Wort sich auch im Mordwinischen findet, muss die Entlehnung vorarabisch sein (denn arabisch نق findet sich als nagat nur sporadisch in Russland).$^{70}$ Nun habe ich schliesslich gefunden, dass in Anatolien ein Königsgrab zur Kubankultur ${ }^{71}$ gehörig aufgefunden ist. Die Kubankultur hat aber wiederum Beziehungen zu der zentralrussischen Fatjanovokultur ${ }^{72}$ gehabt und die assyrischen Kaufleute in Kültepe sind ja nach den Texten wenigstens fast bis zum Schwarzen Meer gekommen, warum konnte dann das Wort sich dann (!) nicht direkt oder indirekt noch nicht (!) weiter ausbreiten? Aber ich denke, dass mir Niemand glauben will, obwohl es auch andere Gemeinsamkeiten zwischen Babylonien und östliches Nordeuropa gibt.

Bei solchem Herumsuchen habe ich so viele sonderbare Sachen gefunden, dass ich manchmal die Kraft von zwanzig Menschen haben möchte, um Alles durchzuarbeiten. Manchmal finde ich sicher etwas schon Gefundenes, aber ich bin schon so müde, dass auch die grösseren »Entdeckungen« mir mehr keine (!) Freude bereiten. Die Indianer und die Polynesier habe ich auch nicht vergessen und es scheint mir, dass ich ein besseres Schema ausarbeiten könnte als die »Kulturkreislehre « von Graebner-Schmidt. ${ }^{73}$ Aber ob ich es jemals zustande bringe, weiss ich nicht und glaube auch nicht. Es wäre zu viel zu tun.

Vielleicht kann ich Ihnen jetzt auch die »1001 Nacht« zurücksenden. Gehört habe ich, dass es möglich ist, noch glaube ich es aber nicht.

\footnotetext{
${ }^{69}$ Hebr. mekes, Zoll, syr. maksā, Zoll (hier translit. in hebräischen Buchstaben, m-ks-'), arab. maks, Zoll, Preis. Vgl. auch Uku Masing, »Akkadisches miksu in Osteuropa«, in: János Harmatta, György Komoróczy (Hrsg.), Wirtschaft und Gesellschaft im alten Vorderasien. Acta Antiqua Academiae Scientarum Hungaricae 22 (1974-1976), 521-526.

70 Arab. naqd, »criticism, critical remark«, aber auch »ready money, cash« (nach Hans Wehr, 4th ed. 1979).

71 Schon im 19. Jahrhundert war die Bezeichnung »Koban-Kultur« üblicher; es handelt sich um eine spätbronze- und eisenzeitliche Kultur vor allem auf der Nordseite des Kaukasus. Ihre Überreste waren 1881 zuerst von Ernest Chantre, Recherches anthropologiques dans la Caucase, 2,1-2: Période protohistorique, Paris 1886 aufgefunden, gesammelt und beschrieben worden und trafen schnell auf internationales Interesse, z. B. Rudolf Virchow, Das Gräberfeld von Koban im Lande der Osseten, Kaukasus: Eine vergleichendarchäologische Studie, Berlin 1883. Die Belegstelle für das genannte Königsgrab in Anatolien bleibt leider obskur.

72 Diese Verbindung wirkt wenig plausibel, da die Fatjanowo-Kultur als jungsteinzeitlich und damit bedeutend älter als die Koban-Kultur angesehen wird, vgl. den Eintrag von James Patrick Mallory, »FatyanovoBalanovo Culture«, in: Encyclopaedia of Indo-European Culture, London 1997, $196 \mathrm{f}$.

${ }^{73}$ Gemeint ist hier die von Fritz Graebner entworfene »Kulturkreislehre« (Methode der Ethnologie, Heidelberg 1911, in Weiterentwicklung der Ideen von Leo Frobenius) und deren Aufnahme durch den Sprachwissenschaftler und Ethnologen P. Wilhelm Schmidt (z. B. in Die Sprachfamilien und Sprachkreise der Erde, Heidelberg 1926).
} 
Und schliesslich, - ich bin Ihnen sehr dankbar für das Versprechen für meine etwaige äthiopistische Arbeiten eine Unterkunft zu finden, vielleicht werde ich Sie bald belästigen. Und noch mehr für Ihre Interesse an meinem Schicksal, denn es gibt nur noch sehr wenige, die daran denken.

J, Hurda 9-3, Tartu, Estland (Ostland)

1.VI 1942
Mit besten Grüssen von mir und von meiner Frau Ihr ergebener + Uku Masing+

\section{8.}

Sehr geehrter Herr Professor,

vielen Dank für Ihren Brief und Ihre Güte mir gegenüber. Wenn es einmal wieder so weit sein sollte, werde ich Sie sicher um eine Empfehlung bitten, aber sobald wird es wohl nicht notwendig sein. Es geht uns irgendwie ziemlich gut, zum Glück braucht man auch heutzutage nicht viel zu wünschen. Und das Porto ist schon vergessen, - ich musste einmal auch die Bücher von der Post zurückholen, weil sie alle zusammen gepackt waren und so zu schwer für Deutschland waren. Ich habe manchmal auch etwas bezahlte Arbeit, wieder eine Geschichte für die Mittelschulen, bekomme für die Paraphrase eine halbe Mark pro Druckseite.

Ich würde Ihnen sehr dankbar für Ihre Arbeiten sein. Was die Bücher anlangt, so habe ich keine Ahnung was seit Mitte 1939 erschienen ist. Wenn ich aber für einige Zeit Kataloge der äthiopischen Sammlungen (ausser Wright, Zotenberg, Conti Rossini (Abbadie), ${ }^{74}$ Rhodokanakis und Ihre eigene ${ }^{75}$ ) bekommen könnte, wäre ich Ihnen sehr dankbar. Leider habe ich Ihnen nichts Eigenes zu schicken. Meine Doktorarbeit existiert in drei Exemplaren, falls Sie Wert darauf legen würde ich Ihnen eine schicken. Aber nur die Universitätsbibliothek hat einen vollständigen gedrucken Exemplar, meine zwei sind maschinenschriftlich ergänzt und teilweise aus den noch vorhandenen Korrekturbogen zusammengesetzt. Sie sehen deshalb nicht sehr schön aus. Ob sie einen anderen Wert haben als nur den der Seltenheit, weiss ich nicht. Ich glaube nicht, dass die Arbeit irgendeinmal neu gedruckt wird und bin auch selbst von ihr schon müde geworden. So habe ich Ihnen fast nichts für Ihre Arbeiten anzubieten. Ich habe nur einige unwichtige hebräische Handschriften, die ich vor dem Kriege aus Dünaburg geholt habe und eine ziemlich alte Ausgabe von »Zohar« (Sulzbach). ${ }^{76}$ Hier ist es ja ziemlich unmöglich sich mit semitischen Sprachen zu beschäftigen.

\footnotetext{
74 Erneut ist Conti Rossini (Anm. 32) gemeint; dieser Katalog war entstanden als Überarbeitung und Erweiterung des Catalogue raisonné des manuscrits éthiopiens, appartenant à Antoine d'Abbadie, Paris 1859, in dem d'Abbadie seine eigene, während eines langjährigen Forschungsaufenthalts (1837-48) in Äthiopien aufgebaute Sammlung erfasst hatte.

75 Murad Kamil, Die abessinischen Handschriften der Sammlung Littmann in Tübingen, Leipzig 1936 (Abhandlungen für die Kunde des Morgenlandes 21, 8).

76 Sefer ha-Zohar, hrsg. Christian Knorr von Rosenroth, Sulzbach 1684. Vgl. Boaz Huss, »Text und Context des Sulzbacher Zohar«, Morgen-Glantz, Zeitschrift der Christian Knorr von Rosenroth-Gesellschaft 16 (2006), 135-159.
} 
Bisher habe ich etwa dreihundert europäische Wunder Mariae verzettelt und etwa zweihundert äthiopische. Bei einer solchen Arbeit ist kaum etwas zu entdecken, da die Angaben über lateinische Legenden nur den Anfang (meistens) geben und ich für die äthiopischen nur eine Kopie der Ausgabe von Budge, die Abschrift einer Berliner Handschrift und die Übersetzungen von Budge habe. Es scheint aber, dass wenigstens für einige äthiopische Texten doch fast wörtlich gleichlautende europäische vorhanden sind, so z. B. für Budge, One hundred and ten Miracles no. LXVII, die Handschrift Paris lat. $18134,49^{\circ}$ von der Mussafia etwas mehr gibt als den Anfang. ${ }^{77}$ Jedenfalls liegt diese lateinische Legende dem äthiopischen Texte näher als acht andere von Poncelet aufgezählte Varianten. Ich hoffe etwa zu Weihnachten mit Mussafia fertig zu werden. Dann aber beginnt erst die Arbeit richtig.

Sonst beschäftige ich mich mit den amerikanischen Sprachen (»Dorpat« ist dazulande eine berühmte Universität gewesen und die grösseren älteren Arbeiten sind deshalb vorhanden) habe eine grammatische Skizze der Bribrisprache geschrieben (vielleicht hat Lehmann ${ }^{78}$ schon eine viel bessere gegeben, aber die kenne ich leider nicht) und arbeite jetzt an den neuen Kičetexten von Leonhard Schultze. ${ }^{79}$ Vielleicht wird mir einmal dadurch auch das semitische Verbum durchsichtiger wenn mir mehrere Möglichkeiten sich auszudrücken bekannt geworden sind. Und zur Erholung versuchen wir, meine Frau mit mir, tibetanisch zu lesen, vergessen was wir gelernt haben und fangen wieder an. Ich habe eine Schwäche für die buddhistische Philosophie und meine Frau für Alexandra David-Neel. ${ }^{80}$

Ich war sehr glücklich als ich Ihren Brief bekam und hoffe, dass ich nicht sehr viel geschwatzt habe. Hoffentlich geht es Ihnen sehr gut, bei uns regnet und regnet es, sodass man beginnt schon traurig zu werden.

J, Hurda 9-3, Tartu

Mit besten Grüssen von meiner Frau und mir

Estland (Ostland)

Ihr ergbener

26.VII 1942

+Uku Masing+

\footnotetext{
77 Mussafia (Anm. 62), I, Wien 1887 (=Sitzungsberichte der phil.-hist. Classe der kais. Akademie der Wissenschaften 113/2 [1886], 917-994), hier: 72 [986]. Die Legende ist vom Typus »Marienbräutigam, Ring am Finger«, im Wesentlichen entsprechend Paris lat. 12593, Nr. 29, jedoch mit abweichendem Anfang, den Masing in Budges Sammlung wiedergefunden zu haben meinte. Ein Jüngling steckt der Marienstatue den Ring seiner Geliebten an den Finger, um ihn vor Beschädigungen während des Ballspiels zu schützen; später wird er Mönch. Der abweichende Beginn der Variante in Paris lat. 18134 berichtet, die Marienstatue sei für eine Almosensammlung vor einer zusammengestürzten Kirche aufgestellt gewesen.

78 Gemeint ist Walter Lehmann, Zentral-Amerika: Teil 1: Die Sprachen Zentral-Amerikas in ihren Beziehungen zueinander sowie zu Süd-Amerika und Mexico, Berlin 1920.

79 Gemeint ist wohl Leonhard Schultze-Jena, Indiana I: Leben, Glaube und Sprache der Quiché von Guatemala, Jena 1933.

80 Alexandra David-Néel (1868-1969), französische Asienreisende, Mitglied der theosophischen Gesellschaft, tibetische Nonne und populärwissenschaftliche Vermittlerin des tibetanischen Buddhismus in Europa; Verbreitung fanden besonders ihr Mystiques et Magiciens du Thibet, Paris 1929 und ihr Reisebericht Voyage d'une Parisienne à Lhassa, à pied et en mendiant de la Chine à l'Inde à travers le Thibet, Paris 1927.
} 


\section{9.}

Sehr geehrter Herr Professor,

besten Dank für Ihren Brief und Ihre Wünsche. Ich hatte ja gar nicht gehofft, dass Sie noch ein Mal mir antworten werden, meine Freude ist ebendeshalb auch gross gewesen. Sonst geht es mir ja nicht so gut, wie es unter den jetzigen Zeitumständen möglich wäre. Es ist schwer zu sagen woran ich mehr gewöhnt bin, an Fieber oder an Fieberlosigkeit, beide wechseln sich so ab, dass ich nicht mehr weiss, welche Lage die normalere sein könnte.

Mit dem Katalogisieren der Marienlegenden bin ich nun so weit wie es hier möglich ist. Ich beabsichtige noch etwas, aber ich weiss nicht, ob diese Arbeit einen Sinn hat. Die semitischen Nomina mit dem präfigierten $\mathrm{m}$ - haben mich immer etwas geärgert, da die Erklärung, dass diese Formen sich aus Nebensätzen gebildet haben, nicht immer passt. Vielleicht könnte man annehmen, dass m- ursprünglich ein Relationspronomen, etwa mit der Bedeutung »das des ...« gewesen ist. Eine solche Ausdrucksweise existiert in indonesischen Sprachen und wäre vielleicht auch in den semitischen Sprachen möglich. Noch weiss ich aber nicht, ob eine solche Theorie sich durchführen lässt, da in einer späteren Phase der Sprachgeschichte sicher mit Analogiebildungen zu rechnen ist. Vielleicht könnten Sie so gut sein und mir Ihre Meinung darüber sagen.

Diejenigen, die an der Verteidigung meiner Doktorarbeit Interesse gehabt haben, scheinen diese verloren zu haben, sie haben andere Sorgen genug. Deshalb will ich auch das Dekanat der Philosophischen Fakultät in Tübingen nicht mit einer Nachfrage belästigen, aber ich werde es tun, sobald man wieder Interesse dafür zeigt. Ich bin aber Ihnen sehr dankbar für die Auskunft und noch mehr dafür, dass ich Ihnen willkommen bin. Das Wetter ist hier wärmer geworden, und ich weiss nicht warum, aber wenn ich jetzt die Sonne sehe, habe ich etwas mehr Hoffnung für alle Menschen und für mich persönlich, obwohl das Letzte sonst gar nicht wichtig scheint.

1.II 1943

J, Hurda 9-3, Tartu

Estland-Ostland
Mit besten Grüssen von mir und meiner Frau

Ihr ergebener + Uku Masing+

Open Access Dieser Artikel wird unter der Creative Commons Namensnennung 4.0 International Lizenz (http://creativecommons.org/licenses/by/4.0/deed.de) veröffentlicht, welche die Nutzung, Vervielfältigung, Bearbeitung, Verbreitung und Wiedergabe in jeglichem Medium und Format erlaubt, sofern Sie den/die ursprünglichen Autor(en) und die Quelle ordnungsgemäß nennen, einen Link zur Creative Commons Lizenz beifügen und angeben, ob Änderungen vorgenommen wurden. 